\title{
Parton distribution functions from nonlocal light-cone operators with definite twist
}

\author{
Bodo Geyer* and Markus Lazar ${ }^{\dagger}$ \\ Center for Theoretical Studies and Institute of Theoretical Physics, \\ Leipzig University, Augustusplatz 10, D-04109 Leipzig, Germany
}

(November 3, 2018)

\begin{abstract}
We introduce the chiral-even and chiral-odd quark distributions as forward matrix elements of related bilocal quark operators with well-defined (geometric) twist. Thereby, we achieve a Lorentz invariant classification of these distributions which differ from the conventional ones by explicitly taking into account the necessary trace terms. The relations between both kinds of distribution functions are given and the mismatch between their different definition of twist is discussed. WandzuraWilczek-like relations between the conventional distributions (based on dynamical twist) are derived by means of geometric twist distribution functions.

PACS number(s): 12.38.Bx; 13.85.Fb
\end{abstract}

\section{INTRODUCTION}

The scattering amplitudes of light-cone dominated hadronic processes - according to the factorization hypothesis are usually represented by the convolution of the hard (process-dependent) scattering amplitude of the partons with appropriate soft (process-independent) parton distribution amplitudes. The different phenomenological distribution amplitudes, e.g., parton distribution functions in deep inelastic scattering and in Drell-Yan processes as well as hadronic wave functions are given as (non-forward) matrix elements of some nonlocal light-cone (LC) operators. Thereby, one and the same LC operator is related to different hadronic processes. Moreover, the classification of these distribution functions suffers from the fact, that these operators by its twist decomposition also contribute to different distribution functions. Therefore, it is necessary to disentangle these various contributions of the original operator and to specify the contributions of the trace terms.

Quite recently, a general group theoretical procedure has been introduced to decompose these nonlocal LC operators [1]:2] into operators of definite twist. This procedure is based on the notion of geometric twist = mass dimension - (Lorentz) spin, $\tau=d-j$, originally introduced by Gross and Treiman [3]. On the other hand, Jaffe and Ji [4] proposed the notion of dynamical twist $(t)$ by counting powers $Q^{2-t}$ which is directly related to the power by which the corresponding distributions contribute to the scattering amplitudes. They found nine independent forward quark distribution amplitudes. Recently, Ball et al. have used this pattern for the consideration of higher twist distribution amplitudes of vector mesons in QCD [5.,6].

However, that dynamical notion of twist is only defined for the matrix elements of operators, it is not Lorentz invariant and also not simply related to the contributions of (higher) geometric twist: Both definitions of twist do not coincide at higher orders. Thus, the definition of dynamical twist has a mismatch with the conventional definition of geometrical twist. This mismatch gives rise to relations of Wandzura-Wilczek type which show that dynamical twist distributions contain various parts of different geometric twist. With the growing accuracy of data for QCD tests, the higher twist contributions become relevant. Therefore, it seems to be quite urgent to consider these contributions on a rigorous quantum field theoretical basis. Jaffe et al. have already pointed out that the geometric twist is the most convenient one to discuss higher twist effects on a reliable basis [4.7. 7 . In addition, it should be emphasized that the LC operators of definite geometric twist are to be preferred also from the point of view of renormalization theory, because they might be assumed to have much simpler renormalization properties then those used in the definition of the conventional structure functions.

The aim of this paper is to present the forward quark distribution functions which are related to the nonlocal LC-operators of different geometric twist. In that framework, it is possible to investigate in an unique manner the

*E-mail: geyer@itp.uni-leipzig.de

${ }^{\dagger}$ E-mail: lazar@itp.uni-leipzig.de 
contributions resulting from the traces of the operators having well-defined twist. By the way, the classification of the various distribution functions appears to be quite straightforward.

The paper is organized as follows. Sect. 2 is mainly introductory. It collects the necessary definitions and explains the basic ideas. Sect. 3 contains a detailed study of chiral-odd as well as chiral-even distribution functions and of the forward matrix elements with definite geometric twist. Whereas the preceeding sections contain the group theoretical aspects the next sections are devoted to the phenomenological aspects. The interrelation between the conventional and the new structure functions of dynamical and geometric twist, respectively, is given in Sect. 4. Thereby, it is proven that both types of structure functions could be used for the parametrization of experimental data because they can be expressed uniquely through each other. However, the virtue of the new structure functions is their well defined twist. This allows also to disentangle the genuine twist content of the conventional distribution functions. This is used in Sect. 5 to derive Wandzura-Wilczek-like relations between the conventional distribution functions. Most of them are new and might be of phenomenological interest. Finally, Sect. 6 contains a summary and conclusions. App. A collects some useful formulae about the interior derivative on the light-cone. Additionally, we rewrite our operators of definite geometric twist by means of this derivative. App. B clarifies some transformation properties of nonlocal operators with definite geometric twist.

\section{TWIST DECOMPOSITION OF BILINEAR QUARK OPERATORS}

At first, we shortly review the systematic procedure of group theoretical twist decomposition for local and nonlocal operators [1.2]. It is based on the unique spin decomposition with respect to the Lorentz group $S O(3,1)$ of the considered operators. The consecutive steps which are used to decompose a bilocal operator, for example, $O_{\alpha}\left(\kappa_{1} x, \kappa_{2} x\right)=\bar{\psi}\left(\kappa_{1} x\right) \gamma^{5} \gamma_{\alpha} U\left(\kappa_{1} x, \kappa_{2} x\right) \psi\left(\kappa_{2} x\right)$, into operators of definite geometric twist are the following:

(1) Taylor expansion of the nonlocal tensor operator for arbitrary values of $x$ into an infinite tower of local tensor operators having rank $n$ and dimension $d$;

(2) Decomposition of these local tensor operators into irreducible ones - with respect to the Lorentz group - by means of the standard method characterizing the symmetry class by Young patterns $[m]=\left(m_{1}, m_{2}, \ldots, m_{r}\right)$ of $r$ rows;

(3) Resummation of the irreducible local tensor operators belonging to the same symmetry class and having the same twist $\tau$ to a nonlocal tensor operator with definite twist;

(4) Projection of the nonlocal twist operator onto the light-cone, $x \rightarrow \tilde{x}$ with $\tilde{x}^{2}=0$, thereby reducing the infinite twist decomposition to a finite one.

In [1] we applied that procedure to the following bilocal quark operators on the light-cone having (axial) vector as well as skew tensor structure:

$$
\begin{aligned}
O_{5 \alpha}\left(\kappa_{1} \tilde{x}, \kappa_{2} \tilde{x}\right) & =\bar{\psi}\left(\kappa_{1} \tilde{x}\right) \gamma^{5} \gamma_{\alpha} U\left(\kappa_{1} \tilde{x}, \kappa_{2} \tilde{x}\right) \psi\left(\kappa_{2} \tilde{x}\right), \\
M_{5[\alpha \beta]}\left(\kappa_{1} \tilde{x}, \kappa_{2} \tilde{x}\right) & =\bar{\psi}\left(\kappa_{1} \tilde{x}\right) \mathrm{i} \gamma^{5} \sigma_{\alpha \beta} U\left(\kappa_{1} \tilde{x}, \kappa_{2} \tilde{x}\right) \psi\left(\kappa_{2} \tilde{x}\right),
\end{aligned}
$$

with the path ordered gauge factor along the straight line connecting the points $\kappa_{1} \tilde{x}$ and $\kappa_{2} \tilde{x}$ :

$$
U\left(\kappa_{1} \tilde{x}, \kappa_{2} \tilde{x}\right)=P \exp \left\{\mathrm{i} g \int_{\kappa_{1}}^{\kappa_{2}} \mathrm{~d} w \tilde{x}^{\mu} A_{\mu}(w \tilde{x})\right\},
$$

and to the corresponding operators without $\gamma_{5}$ and the derived scalar and vector operators, $O_{(5)}\left(\kappa_{1} \tilde{x}, \kappa_{2} \tilde{x}\right)=$ $x^{\alpha} O_{(5) \alpha}\left(\kappa_{1} \tilde{x}, \kappa_{2} \tilde{x}\right)$ and $M_{(5) \alpha}\left(\kappa_{1} \tilde{x}, \kappa_{2} \tilde{x}\right)=x^{\beta} M_{(5)[\alpha \beta]}\left(\kappa_{1} \tilde{x}, \kappa_{2} \tilde{x}\right)$, respectively.

The resulting decomposition for the vector and skew tensor operators - which is independent of the presence or absence of $\gamma_{5}$ - after a straightforward calculation is:

$$
\begin{aligned}
O_{\alpha}\left(\kappa_{1} \tilde{x}, \kappa_{2} \tilde{x}\right) & =O_{\alpha}^{\mathrm{tw} 2}\left(\kappa_{1} \tilde{x}, \kappa_{2} \tilde{x}\right)+O_{\alpha}^{\mathrm{tw} 3}\left(\kappa_{1} \tilde{x}, \kappa_{2} \tilde{x}\right)+O_{\alpha}^{\mathrm{tw} 4}\left(\kappa_{1} \tilde{x}, \kappa_{2} \tilde{x}\right), \\
M_{[\alpha \beta]}\left(\kappa_{1} \tilde{x}, \kappa_{2} \tilde{x}\right) & =M_{[\alpha \beta]}^{\mathrm{tw} 2}\left(\kappa_{1} \tilde{x}, \kappa_{2} \tilde{x}\right)+M_{[\alpha \beta]}^{\mathrm{tw} 3}\left(\kappa_{1} \tilde{x}, \kappa_{2} \tilde{x}\right)+M_{[\alpha \beta]}^{\mathrm{tw} 4}\left(\kappa_{1} \tilde{x}, \kappa_{2} \tilde{x}\right), \\
M_{\alpha}\left(\kappa_{1} \tilde{x}, \kappa_{2} \tilde{x}\right) & =M_{\alpha}^{\mathrm{tw} 2}\left(\kappa_{1} \tilde{x}, \kappa_{2} \tilde{x}\right)+M_{\alpha}^{\mathrm{tw} 3}\left(\kappa_{1} \tilde{x}, \kappa_{2} \tilde{x}\right) .
\end{aligned}
$$


with (using the convention $\left.a_{[\mu} b_{\nu]} \equiv(1 / 2)\left(a_{\mu} b_{\nu}-a_{\nu} b_{\mu}\right)\right)$

$$
\begin{aligned}
& O^{\mathrm{tw} 2}\left(\kappa_{1} \tilde{x}, \kappa_{2} \tilde{x}\right)=\tilde{x}^{\mu} O_{\mu}\left(\kappa_{1} \tilde{x}, \kappa_{2} \tilde{x}\right)=\bar{\psi}\left(\kappa_{1} \tilde{x}\right)(\gamma \tilde{x}) U\left(\kappa_{1} \tilde{x}, \kappa_{2} \tilde{x}\right) \psi\left(\kappa_{2} \tilde{x}\right) \\
& O_{\alpha}^{\mathrm{tw} 2}\left(\kappa_{1} \tilde{x}, \kappa_{2} \tilde{x}\right)=\left.\int_{0}^{1} \mathrm{~d} \lambda\left(\partial_{\alpha}+\frac{1}{2}(\ln \lambda) x_{\alpha} \square\right) x^{\mu} O_{\mu}\left(\kappa_{1} \lambda x, \kappa_{2} \lambda x\right)\right|_{x=\tilde{x}} \\
& O_{\alpha}^{\mathrm{tw} 3}\left(\kappa_{1} \tilde{x}, \kappa_{2} \tilde{x}\right)=\left.\int_{0}^{1} \mathrm{~d} \lambda\left(\delta_{\alpha}^{\mu}(x \partial)-x^{\mu} \partial_{\alpha}-(1+2 \ln \lambda) x_{\alpha} \partial^{\mu}-(\ln \lambda) x_{\alpha} x^{\mu} \square\right) O_{\mu}\left(\kappa_{1} \lambda x, \kappa_{2} \lambda x\right)\right|_{x=\tilde{x}} \\
& O_{\alpha}^{\mathrm{tw} 4}\left(\kappa_{1} \tilde{x}, \kappa_{2} \tilde{x}\right)=\left.\tilde{x}_{\alpha} \int_{0}^{1} \mathrm{~d} \lambda\left((1+\ln \lambda) \partial^{\mu}+\frac{1}{2}(\ln \lambda) x^{\mu} \square\right) O_{\mu}\left(\kappa_{1} \lambda x, \kappa_{2} \lambda x\right)\right|_{x=\tilde{x}} \\
& M_{[\alpha \beta]}^{\mathrm{tw} 2}\left(\kappa_{1} \tilde{x}, \kappa_{2} \tilde{x}\right)=\left.\int_{0}^{1} \mathrm{~d} \lambda\left\{2 \lambda \partial_{[\beta} \delta_{\alpha]}^{\mu}-(1-\lambda)\left(2 x_{[\alpha} \partial_{\beta]} \partial^{\mu}-x_{[\alpha} \delta_{\beta]}^{\mu} \square\right)\right\} x^{\nu} M_{[\mu \nu]}\left(\kappa_{1} \lambda x, \kappa_{2} \lambda x\right)\right|_{x=\tilde{x}} \\
& M_{[\alpha \beta]}^{\mathrm{tw} 3}\left(\kappa_{1} \tilde{x}, \kappa_{2} \tilde{x}\right)=\int_{0}^{1} \mathrm{~d} \lambda\left\{\lambda\left((x \partial) \delta_{[\beta}^{\nu}-2 x^{\nu} \partial_{[\beta}\right) \delta_{\alpha]}^{\mu}+\frac{1-\lambda^{2}}{\lambda}\left(x_{[\alpha}\left(\delta_{\beta]}^{[\mu}(x \partial)-x^{[\mu} \partial_{\beta]}\right) \partial^{\nu]}-x_{[\alpha} \delta_{\beta]}^{[\mu} x^{\nu]}\right.\right. \\
& M_{[\alpha \beta]}^{\mathrm{tw} 4}\left(\kappa_{1} \tilde{x}, \kappa_{2} \tilde{x}\right)=\left.\int_{0}^{1} \mathrm{~d} \lambda \frac{1-\lambda}{\lambda}\left\{x_{[\alpha} \delta_{\beta]}^{[\mu} x^{\nu]} \square-2 x_{[\alpha}\left(\delta_{\beta]}^{[\mu}(x \partial)-x^{[\mu} \partial_{\beta]}\right) \partial^{\nu]}\right\} M_{[\mu \nu]}\left(\kappa_{1} \lambda x, \kappa_{2} \lambda x\right)\right|_{x=\tilde{x}} \\
& M_{\alpha}^{\mathrm{tw} 2}\left(\kappa_{1} \tilde{x}, \kappa_{2} \tilde{x}\right)=M_{\alpha}\left(\kappa_{1} \tilde{x}, \kappa_{2} \tilde{x}\right)-\left.\tilde{x}_{\alpha} \partial^{\mu} \int_{0}^{1} \mathrm{~d} \lambda \lambda M_{\mu}\left(\kappa_{1} \lambda x, \kappa_{2} \lambda x\right)\right|_{x=\tilde{x}} \\
& M_{\alpha}^{\mathrm{tw} 3}\left(\kappa_{1} \tilde{x}, \kappa_{2} \tilde{x}\right)=\left.\tilde{x}_{\alpha} \partial^{\mu} \int_{0}^{1} \mathrm{~d} \lambda \lambda M_{\mu}\left(\kappa_{1} \lambda x, \kappa_{2} \lambda x\right)\right|_{x=\tilde{x}} .
\end{aligned}
$$

Obviously, the trace terms are those being multiplied by $\tilde{x}^{\alpha}$ (or $\tilde{x}^{\beta}$ ).

Let us remark that the (axial) vector and skew tensor operators of twist $\tau, O_{(5) \alpha}^{(\tau)}\left(\kappa_{1} \tilde{x}, \kappa_{2} \tilde{x}\right)$ and $M_{(5)[\alpha \beta]}^{(\tau)}\left(\kappa_{1} \tilde{x}, \kappa_{2} \tilde{x}\right)$, are obtained from the (undecomposed) operators $O_{(5) \alpha}\left(\kappa_{1} \tilde{x}, \kappa_{2} \tilde{x}\right)$ and $M_{(5)[\alpha \beta]}\left(\kappa_{1} \tilde{x}, \kappa_{2} \tilde{x}\right)$, Eqs. 2.1]) and (2.2), by the application of the corresponding (complete set of) orthogonal projectors (including the $\lambda$-integrations), $\mathcal{P}_{\alpha}^{(\tau) \mu}$ and $\mathcal{P}_{[\alpha \beta]}^{(\tau)[\mu \nu]}$, defined by Eqs. (2.8) - (2.10) and (2.11) - 2.13), respectively:

$$
\begin{aligned}
O_{(5) \alpha}^{(\tau)}\left(\kappa_{1} \tilde{x}, \kappa_{2} \tilde{x}\right) & =\left(\mathcal{P}_{\alpha}^{(\tau) \mu} O_{(5) \mu}\right)\left(\kappa_{1} \tilde{x}, \kappa_{2} \tilde{x}\right) \\
M_{(5)[\alpha \beta]}^{(\tau)}\left(\kappa_{1} \tilde{x}, \kappa_{2} \tilde{x}\right) & =\left(\mathcal{P}_{[\alpha \beta]}^{(\tau)[\mu \nu]} M_{(5)[\mu \nu]}\right)\left(\kappa_{1} \tilde{x}, \kappa_{2} \tilde{x}\right) \\
M_{(5) \alpha}^{(\tau)}\left(\kappa_{1} \tilde{x}, \kappa_{2} \tilde{x}\right) & =\left(\mathcal{P}_{(v) \alpha}^{(\tau) \mu} M_{(5) \mu}\right)\left(\kappa_{1} \tilde{x}, \kappa_{2} \tilde{x}\right),
\end{aligned}
$$

thereby observing

$$
\tilde{x}^{\alpha} M_{(5) \alpha}\left(\kappa_{1} \tilde{x}, \kappa_{2} \tilde{x}\right)=0
$$

with

$$
\begin{gathered}
\left(\mathcal{P}^{(\tau)} \times \mathcal{P}^{\left(\tau^{\prime}\right)}\right)_{\alpha}^{\mu}=\delta^{\tau \tau^{\prime}} \mathcal{P}_{\alpha}^{(\tau) \mu} \\
\left(\mathcal{P}^{(\tau)} \times \mathcal{P}^{\left(\tau^{\prime}\right)}\right)_{[\alpha \beta]}^{[\mu \nu]}=\delta^{\tau \tau^{\prime}} \mathcal{P}_{[\alpha \beta]}^{(\tau)[\mu \nu]} .
\end{gathered}
$$

These projection properties are due to the fact that the Young operators for different symmetry classes are orthogonal projections on the tensor space. For hints concerning the proof we refer to the Appendix.

In addition, we point to the fact that the twist-2 vector and skew tensor operators, Eqs. (2.8) and (2.11), are related to the corresponding scalar and vector operators, (2.7) and (2.14), respectively. This leads to relations of the corresponding distribution functions, for example, cf. Eqs. (3.2) and (3.4) below.

For convenience, we give the corresponding local expressions of the nonlocal operators, Eqs. (2.7) - (2.15). The relation between the local and nonlocal operators are $\left(\kappa_{1}=0\right)$

$$
O_{\alpha}\left(0, \kappa_{2} \tilde{x}\right)=\sum_{n=0}^{\infty} \frac{\kappa_{2}^{n}}{n !} O_{\alpha n}(\tilde{x}), \quad M_{[\alpha \beta]}\left(0, \kappa_{2} \tilde{x}\right)=\sum_{n=0}^{\infty} \frac{\kappa_{2}^{n}}{n !} M_{[\alpha \beta] n}(\tilde{x})
$$


The expressions for the local operators are

$$
\begin{aligned}
O_{n+1}^{\mathrm{tw} 2}(\tilde{x}) & \equiv \tilde{x}^{\mu} O_{\mu n}(\tilde{x})=\bar{\psi}(0) \gamma^{5}(\gamma \tilde{x})(\tilde{x} D)^{n} \psi(0) \\
O_{\alpha n}^{\mathrm{tw} 2}(\tilde{x}) & =\left.\frac{1}{n+1}\left(\partial_{\alpha}-\frac{1}{2(n+1)} x_{\alpha} \square\right) O_{n+1}(x)\right|_{x=\tilde{x}} \\
O_{\alpha n}^{\mathrm{tw} 3}(\tilde{x}) & =\left.\frac{1}{n+1}\left(n \delta_{\alpha}^{\mu}-x^{\mu} \partial_{\alpha}-\frac{1}{n+1} x_{\alpha}\left((n-1) \partial^{\mu}-x^{\mu} \square\right)\right) O_{\mu n}(x)\right|_{x=\tilde{x}} \\
O_{\alpha n}^{\mathrm{tw} 4}(\tilde{x}) & =\left.\frac{1}{(n+1)^{2}} \tilde{x}_{\alpha}\left(n \partial^{\mu}-\frac{1}{2} x^{\mu} \square\right) O_{\mu n}(x)\right|_{x=\tilde{x}} \\
M_{[\alpha \beta] n}^{\mathrm{tw} 2}(\tilde{x}) & =\left.\frac{1}{n+2}\left\{2 \partial_{[\beta} \delta_{\alpha]}^{\mu}-\frac{1}{n+1}\left(2 x_{[\alpha} \partial_{\beta]} \partial^{\mu}-x_{[\alpha} \delta_{\beta]}^{\mu} \square\right)\right\} M_{\mu n+1}(x)\right|_{x=\tilde{x}} \\
M_{[\alpha \beta] n}^{\mathrm{tw} 3}(\tilde{x}) & =\frac{1}{n+2}\left\{\left(n \delta_{[\beta}^{\nu}-2 x^{\nu} \partial_{[\beta}\right) \delta_{\alpha]}^{\mu}+\frac{2}{n}\left(x_{[\alpha}\left((n-1) \delta_{\beta]}^{[\mu}-x^{[\mu} \partial_{\beta]}\right) \partial^{\nu]}-x_{[\alpha} \delta_{\beta]}^{[\mu} x^{\nu]} \square\right.\right. \\
M_{[\alpha \beta] n}^{\mathrm{tw} 4}(\tilde{x}) & \left.\left.=\frac{1}{(n+1) n}\left\{x_{[\alpha} \partial_{\beta]}^{[\mu} x_{\beta]}^{[\mu} \partial^{\nu]}\right)\right\}\left.M_{[\mu \nu] n}(x)\right|_{x=\tilde{x}}-2 x_{[\alpha}\left((n-1) \delta_{\beta]}^{[\mu}-x^{[\mu} \partial_{\beta]}\right) \partial^{\nu]}\right\}\left.M_{[\mu \nu] n}(x)\right|_{x=\tilde{x}} \\
M_{\alpha n+1}^{\mathrm{tw} 2}(\tilde{x}) & =M_{\alpha n+1}(\tilde{x})-\left.\frac{1}{n+2} \tilde{x}_{\alpha} \partial^{\mu} M_{\mu n+1}(x)\right|_{x=\tilde{x}} \\
M_{\alpha n+1}^{\mathrm{tw} 3}(\tilde{x}) & =\left.\frac{1}{n+2} \tilde{x}_{\alpha} \partial^{\mu} M_{\mu n+1}(x)\right|_{x=\tilde{x}} .
\end{aligned}
$$

Obviously, analogous projection properties as mentioned above for the nonlocal operators also hold for the local ones. Let us further remark that the projection properties of the above nonlocal and local LC operators as well as the conditions of their tracelessness can be formulated by means of inner derivatives on the light-cone (see Appendix A).

\section{FORWARD MATRIX ELEMENTS OF LC-OPERATORS WITH TWIST $\tau$}

Now, we define the (polarized) quark distribution functions for the bilinear quark operators with definite twist. As usual, the matrix elements of the nucleon targets, $\bar{u}(P, S) \gamma^{\mu} u(P, S)=2 P^{\mu}$ and $\bar{u}(P, S) \gamma^{5} \gamma^{\mu} u(P, S)=2 S^{\mu}$, are related to the nucleon momentum $P_{\mu}$ and nucleon spin vector $S_{\mu}$, respectively, with $P^{2}=M^{2}, S^{2}=-M^{2}, P \cdot S=0, M$ denoting the nucleon mass. Here $u(P, S)$ denotes the free hadronic spinor.

Taking forward matrix elements of Eqs. (2.7) - (2.15) we see that, observing the correct tensor structure by the use of $P_{\mu}, S_{\mu}$ and $\tilde{x}_{\mu}$, we may introduce any parametrization for the matrix elements of the undecomposed operators, e.g.,

$$
\left\langle P S\left|O_{(5) \alpha}\left(\kappa_{1} \tilde{x}, \kappa_{2} \tilde{x}\right)\right| P S\right\rangle=2 \int_{0}^{1} \mathrm{~d} z\left(S_{\alpha} \widetilde{G}_{1}\left(z, \mu^{2}\right)+P_{\alpha} \frac{\tilde{x} S}{\tilde{x} P} \widetilde{G}_{2}\left(z, \mu^{2}\right)+\tilde{x}_{\alpha} \frac{\tilde{x} S}{(\tilde{x} P)^{2}} M^{2} \widetilde{G}_{3}\left(z, \mu^{2}\right)\right) \mathrm{e}^{\mathrm{i} \kappa z(\tilde{x} P)},
$$

where, $\mu^{2}$ denotes the renormalization scale and, also in the following, we defined $\kappa=\kappa_{1}-\kappa_{2}$. Such a choice is not preferable because the matrix element of any twist- $\tau$ operator then depends in a complicated way from every distribution function. However, according to the above projection properties we are able to introduce one (and only one) distribution function for any operator of definite twist. For cases where the local twist $-\tau$ operators are already known in the literature that choice leads to the same result.

Let us first consider the chiral-even pseudo scalar operator in the polarized case. The (forward) matrix element of this twist-2 operator, Eq. (2.7), taken between hadron states $|P S\rangle$ is trivially represented as

$$
\left\langle P S\left|O_{5}^{\mathrm{tw} 2}\left(\kappa_{1} \tilde{x}, \kappa_{2} \tilde{x}\right)\right| P S\right\rangle=2(\tilde{x} S) \int_{0}^{1} \mathrm{~d} z G^{(2)}\left(z, \mu^{2}\right) \mathrm{e}^{\mathrm{i} \kappa z(\tilde{x} P)}=2(\tilde{x} S) \sum_{n=0}^{\infty} \frac{(\mathrm{i} \kappa(\tilde{x} P))^{n}}{n !} G_{n}^{(2)}\left(\mu^{2}\right) .
$$

Here, $G^{(2)}\left(z, \mu^{2}\right)$ is the uniquely defined twist-2 parton distribution function which by a Mellin transformation is obtained from the corresponding moments

$$
G_{n}^{(2)}\left(\mu^{2}\right)=\int_{0}^{1} \mathrm{~d} z z^{n} G^{(2)}\left(z, \mu^{2}\right)
$$

The nonforward matrix elements of this scalar operator, Eq. 2.7), are discussed in 911 . 
Now we consider the chiral-even axial vector operator. Because of the projection properties (2.16), together with (2.19), we introduce the parton distribution functions $G^{(\tau)}\left(z, \mu^{2}\right)$ of twist $\tau$ by

$$
\left\langle P S\left|O_{5 \alpha}^{(\tau)}\left(\kappa_{1} \tilde{x}, \kappa_{2} \tilde{x}\right)\right| P S\right\rangle \equiv\left\langle P S\left|\left(\mathcal{P}_{\alpha}^{(\tau) \beta} O_{5 \beta}^{(\tau)}\right)\left(\kappa_{1} \tilde{x}, \kappa_{2} \tilde{x}\right)\right| P S\right\rangle=\mathcal{P}_{\alpha}^{(\tau) \beta}\left(2 S_{\beta} \int_{0}^{1} \mathrm{~d} z G^{(\tau)}\left(z, \mu^{2}\right) \mathrm{e}^{\mathrm{i} \kappa z(\tilde{x} P)}\right)
$$

For $\tau=2$ this is consistent with $(3.2)$, it reads

$$
\left\langle P S\left|O_{5 \alpha}^{\mathrm{tw} 2}\left(\kappa_{1} \tilde{x}, \kappa_{2} \tilde{x}\right)\right| P S\right\rangle=\left.2 \int_{0}^{1} \mathrm{~d} \lambda\left[\partial_{\alpha}+\frac{1}{2} \ln (\lambda) x_{\alpha} \square\right](x S) \int_{0}^{1} \mathrm{~d} z G^{(2)}\left(z, \mu^{2}\right) \mathrm{e}^{\mathrm{i} \kappa \lambda z(x P)}\right|_{x=\tilde{x}} .
$$

Using the projection operators as they are determined by Eqs. $(2.8)-(2.10)$ we obtain (from now on we suppress $\mu^{2}$ )

$$
\begin{aligned}
\left\langle P S\left|O_{5 \alpha}^{\mathrm{tw} 2}\left(\kappa_{1} \tilde{x}, \kappa_{2} \tilde{x}\right)\right| P S\right\rangle & =2 \int_{0}^{1} \mathrm{~d} \lambda \int_{0}^{1} \mathrm{~d} z G^{(2)}(z)\left[S_{\alpha}+\mathrm{i} \kappa \lambda z P_{\alpha}(\tilde{x} S)+\frac{\tilde{x}_{\alpha}}{2}(\tilde{x} S) M^{2}(\mathrm{i} \kappa \lambda z)^{2}(\ln \lambda)\right] \mathrm{e}^{\mathrm{i} \kappa \lambda z(\tilde{x} P)} \\
& =2 \sum_{n=0}^{\infty} \frac{(\mathrm{i} \kappa(\tilde{x} P))^{n}}{n !} G_{n}^{(2)}\left\{\frac{1}{n+1}\left(S_{\alpha}+n P_{\alpha} \frac{\tilde{x} S}{\tilde{x} P}\right)-\frac{n(n-1)}{2(n+1)^{2}} \tilde{x}_{\alpha} \frac{\tilde{x} S}{(\tilde{x} P)^{2}} M^{2}\right\} \\
\left\langle P S\left|O_{5 \alpha}^{\mathrm{tw} 3}\left(\kappa_{1} \tilde{x}, \kappa_{2} \tilde{x}\right)\right| P S\right\rangle & =2 \int_{0}^{1} \mathrm{~d} \lambda \int_{0}^{1} \mathrm{~d} z G^{(3)}(z)\left[\left(S_{\alpha}(\tilde{x} P)-P_{\alpha}(\tilde{x} S)\right) \mathrm{i} \kappa \lambda z-\tilde{x}_{\alpha} M^{2}(\tilde{x} S)(\mathrm{i} \kappa \lambda z)^{2} \ln \lambda\right] \mathrm{e}^{\mathrm{i} \kappa \lambda z(\tilde{x} P)} \\
& =2 \sum_{n=1}^{\infty} \frac{(\mathrm{i} \kappa(\tilde{x} P))^{n}}{n !} G_{n}^{(3)}\left\{\frac{n}{n+1}\left(S_{\alpha}-P_{\alpha} \frac{\tilde{x} S}{\tilde{x} P}\right)+\frac{n(n-1)}{(n+1)^{2}} \tilde{x}_{\alpha} \frac{\tilde{x} S}{(\tilde{x} P)^{2}} M^{2}\right\} \\
\left\langle P S\left|O_{5 \alpha}^{\mathrm{tw} 4}\left(\kappa_{1} \tilde{x}, \kappa_{2} \tilde{x}\right)\right| P S\right\rangle & =\int_{0}^{1} \mathrm{~d} \lambda \int_{0}^{1} \mathrm{~d} z G^{(4)}(z)\left[\tilde{x}_{\alpha}(\tilde{x} S) M^{2}(\mathrm{i} \kappa \lambda z)^{2} \ln \lambda\right] \mathrm{e}^{\mathrm{i} \kappa \lambda z(\tilde{x} P)} \\
& =-\sum_{n=2}^{\infty} \frac{(\mathrm{i} \kappa(\tilde{x} P))^{n}}{n !} G_{n}^{(4)} \frac{n(n-1)}{(n+1)^{2}} \tilde{x}_{\alpha} \frac{\tilde{x} S}{(\tilde{x} P)^{2}} M^{2} .
\end{aligned}
$$

In the first line of any equation we have written the nonlocal LC-operators of definite twist, and in the second line, after expanding the exponential, we introduced the moments of the structure functions; thereby, the $\lambda$-integrations contribute the additional $n$-dependent factors. The local twist- 2 and twist- 3 matrix elements of traceless operators are given off-cone in Refs. [12,13 for $n \leq 3$ and in Refs. [14,15 for any $n$. Obviously, the trace terms which have been explicitly subtracted are proportional to $M^{2}$. According to the terminology of Jaffe and Ji, they contribute to dynamical twist-4. For the twist-2 operator we observe that the terms proportional to $S_{\alpha}, P_{\alpha}$ and $\tilde{x}_{\alpha}$ have contributions starting with the zeroth, first and second moment, respectively. The twist- 3 operator starts with the first moment, and the twist-4 operator starts with the second moment. Analogous statements also hold for the twist- $\tau$ operators below.

Putting together the different twist contributions we obtain, after replacing i $\kappa \lambda z(\tilde{x} P)$ by $\lambda \partial / \partial \lambda$ and performing partial integrations, the following forward matrix element of the original operator $(\zeta=\kappa(\tilde{x} P))$

$$
\begin{aligned}
\left\langle P S\left|O_{5 \alpha}\left(\kappa_{1} \tilde{x}, \kappa_{2} \tilde{x}\right)\right| P S\right\rangle & =2 P_{\alpha} \frac{\tilde{x} S}{\tilde{x} P} \int_{0}^{1} \mathrm{~d} z\left(G^{(2)}(z)-G^{(3)}(z)\right)\left[e_{0}(\mathrm{i} \zeta z)-e_{1}(\mathrm{i} \zeta z)\right] \\
& +2 S_{\alpha} \int_{0}^{1} \mathrm{~d} z\left(G^{(2)}(z) e_{1}(\mathrm{i} \zeta z)+G^{(3)}(z)\left[e_{0}(\mathrm{i} \zeta z)-e_{1}(\mathrm{i} \zeta z)\right]\right) \\
& -\tilde{x}_{\alpha} \frac{\tilde{x} S}{(\tilde{x} P)^{2}} M^{2} \int_{0}^{1} \mathrm{~d} z\left(G^{(2)}(z)-2 G^{(3)}(z)+G^{(4)}(z)\right)\left[e_{0}(\mathrm{i} \zeta z)-3 e_{1}(\mathrm{i} \zeta z)+2 \int_{0}^{1} \mathrm{~d} \lambda e_{1}(\mathrm{i} \zeta \lambda z)\right]
\end{aligned}
$$

where we introduced the following "truncated exponentials"

$$
e_{0}(\mathrm{i} \zeta z)=\mathrm{e}^{\mathrm{i} \zeta z}, \quad e_{1}(\mathrm{i} \zeta z)=\int_{0}^{1} \mathrm{~d} \lambda \mathrm{e}^{\mathrm{i} \zeta z \lambda}=\frac{\mathrm{e}^{\mathrm{i} \zeta z}-1}{\mathrm{i} \zeta z}, \quad \cdots \quad, e_{n+1}(\mathrm{i} \zeta z)=\frac{(-1)^{n}}{n !} \int_{0}^{1} \mathrm{~d} \lambda \lambda^{n} \mathrm{e}^{\mathrm{i} \zeta z \lambda} .
$$

As it should be the application of the projection operators $\mathcal{P}_{\alpha}^{(\tau) \beta}$ onto $(3.10)$ reproduces the matrix elements $(3.4)-$ (3.8). In comparison with (3.1) we also observe that the distribution functions are accompanied not simply by the exponentials, $e_{0}(\mathrm{i} \zeta z)$, but by more involved combinations whose series expansion directly leads to the representations with the help of moments. 
Now we consider the chiral-even vector operator $O_{\alpha}\left(\kappa_{1} \tilde{x}, \kappa_{2} \tilde{x}\right)=\bar{\psi}\left(\kappa_{1} \tilde{x}\right) \gamma_{\alpha} U\left(\kappa_{1} \tilde{x}, \kappa_{2} \tilde{x}\right) \psi\left(\kappa_{2} \tilde{x}\right)$, which obeys relations Eqs. (2.7) - (2.10), as well as (2.16) and (2.19) with the same projection operator as the axial vector operator. Let us introduce the corresponding parton distribution functions $F^{(\tau)}(z)$ of twist $\tau$ by

$$
\left\langle P S\left|O_{\alpha}^{(\tau)}\left(\kappa_{1} \tilde{x}, \kappa_{2} \tilde{x}\right)\right| P S\right\rangle=\mathcal{P}_{\alpha}^{(\tau) \mu}\left(2 P_{\mu} \int_{0}^{1} \mathrm{~d} z F^{(\tau)}(z) \mathrm{e}^{\mathrm{i} \kappa z(x P)}\right) .
$$

Again, this is consistent with the definition of the unpolarized distribution function $F^{(2)}(z)$ by the matrix element of the corresponding twist-2 scalar operator

$$
\left\langle P\left|O^{\mathrm{tw} 2}\left(\kappa_{1} \tilde{x}, \kappa_{2} \tilde{x}\right)\right| P\right\rangle=2(\tilde{x} P) \int_{0}^{1} \mathrm{~d} z F^{(2)}(z) \mathrm{e}^{\mathrm{i} \kappa z(\tilde{x} P)}=2(\tilde{x} P) \sum_{n=0}^{\infty} \frac{(\mathrm{i} \kappa(\tilde{x} P))^{n}}{n !} F_{n}^{(2)} .
$$

The nonforward matrix elements of this scalar operator has been considered for the first time in [18].

The forward matrix elements of the vector operators of twist $\tau$ are obtained as follows:

$$
\begin{aligned}
\left\langle P\left|O_{\alpha}^{\mathrm{tw} 2}\left(\kappa_{1} \tilde{x}, \kappa_{2} \tilde{x}\right)\right| P\right\rangle & =2 \int_{0}^{1} \mathrm{~d} \lambda \int_{0}^{1} \mathrm{~d} z F^{(2)}(z)\left[P_{\alpha}(1+\mathrm{i} \kappa \lambda z(\tilde{x} P))+\tilde{x}_{\alpha} M^{2} \mathrm{i} \kappa \lambda z \ln \lambda\left(1+\frac{1}{2} \mathrm{i} \kappa \lambda z(\tilde{x} P)\right)\right] \mathrm{e}^{\mathrm{i} \kappa \lambda z(\tilde{x} P)} \\
& =2 \sum_{n=0}^{\infty} \frac{(\mathrm{i} \kappa(\tilde{x} P))^{n}}{n !} F_{n}^{(2)}\left\{P_{\alpha}-\frac{n}{2(n+1)} \tilde{x}_{\alpha} \frac{M^{2}}{\tilde{x} P}\right\}, \\
\left\langle P\left|O_{\alpha}^{\mathrm{tw} 3}\left(\kappa_{1} \tilde{x}, \kappa_{2} \tilde{x}\right)\right| P\right\rangle & =-2 \tilde{x}_{\alpha} M^{2} \int_{0}^{1} \mathrm{~d} \lambda \int_{0}^{1} \mathrm{~d} z F^{(3)}(z) \mathrm{i} \kappa \lambda z[(1+2 \ln \lambda)+\mathrm{i} \kappa \lambda z(\ln \lambda)(\tilde{x} P)] \mathrm{e}^{\mathrm{i} \kappa \lambda z(\tilde{x} P)} \equiv 0, \\
\left\langle P\left|O_{\alpha}^{\mathrm{tw} 4}\left(\kappa_{1} \tilde{x}, \kappa_{2} \tilde{x}\right)\right| P\right\rangle & =-2 \tilde{x}_{\alpha} M^{2} \int_{0}^{1} \mathrm{~d} \lambda \int_{0}^{1} \mathrm{~d} z F^{(4)}(z) \mathrm{i} \kappa \lambda z(\ln \lambda)\left(1+\frac{1}{2} \mathrm{i} \kappa \lambda z(\tilde{x} P)\right) \mathrm{e}^{\mathrm{i} \kappa \lambda z(\tilde{x} P)} \\
& =\sum_{n=1}^{\infty} \frac{(\mathrm{i} \kappa(\tilde{x} P))^{n}}{n !} F_{n}^{(4)} \frac{n}{(n+1)} \tilde{x}_{\alpha} \frac{M^{2}}{\tilde{x} P},
\end{aligned}
$$

The off-cone traceless local twist-2 matrix element has already been obtained in 16, 17. The vanishing of the twist-3 structure function $F^{(3)}(z)$ which follows here by partial integration proves in the nonlocal case the same fact that Jaffe and Soldate have already proved for the local twist-3 operator [7]. The trace terms of the twist-2 operator and the twist- 4 operator itself both contribute to dynamical twist-4 and, consequently, to the same $1 / Q^{2}$-behaviour in the cross section.

Putting together all the contributions of different twist and performing partial $\lambda$-integrations, we obtain the following representation for the forward matrix element of the original operator

$$
\left\langle P\left|O_{\alpha}\left(\kappa_{1} \tilde{x}, \kappa_{2} \tilde{x}\right)\right| P\right\rangle=2 P_{\alpha} \int_{0}^{1} \mathrm{~d} z F^{(2)}(z) e_{0}(\mathrm{i} \zeta z)-\tilde{x}_{\alpha} \frac{M^{2}}{\tilde{x} P} \int_{0}^{1} \mathrm{~d} z\left(F^{(2)}(z)-F^{(4)}(z)\right)\left[e_{0}(\mathrm{i} \zeta z)-e_{1}(\mathrm{i} \zeta z)\right] .
$$

The matrix element of the simplest bilocal scalar operator arises as

$$
\left\langle P\left|\bar{\psi}\left(\kappa_{1} \tilde{x}\right) U\left(\kappa_{1} \tilde{x}, \kappa_{2} \tilde{x}\right) \psi\left(\kappa_{2} \tilde{x}\right)\right| P\right\rangle=2 M \int_{0}^{1} \mathrm{~d} z E^{(3)}(z) \mathrm{e}^{\mathrm{i} \kappa z(\tilde{x} P)}=2 M \sum_{n=0}^{\infty} \frac{(\mathrm{i} \kappa(\tilde{x} P))^{n}}{n !} E_{n}^{(3)},
$$

where $E^{(3)}(z)$ is another spin-independent twist-3 structure function.

Now, we consider the matrix elements of the chiral-odd vector operators. In fact they may be obtained from the chiral-odd skew-tensor operator by contraction with $\tilde{x}_{\beta}$ (cf. Eq. (2.6)). This also determines the corresponding structure functions which are introduced by

$$
\left\langle P S\left|M_{5[\alpha \beta]}^{(\tau)}\left(\kappa_{1} \tilde{x}, \kappa_{2} \tilde{x}\right)\right| P S\right\rangle=\frac{2}{M} \mathcal{P}_{[\alpha \beta]}^{(\tau)[\mu \nu]}\left(\left(S_{\mu} P_{\nu}-S_{\nu} P_{\mu}\right) \int_{0}^{1} \mathrm{~d} z H^{(\tau)}(z) \mathrm{e}^{\mathrm{i} \kappa z(\tilde{x} P)}\right) .
$$


Applying the same procedure as above we obtain

$$
\begin{aligned}
\left\langle P S\left|M_{5 \alpha}^{\mathrm{tw} 2}\left(\kappa_{1} \tilde{x}, \kappa_{2} \tilde{x}\right)\right| P S\right\rangle & =\frac{2}{M} \int_{0}^{1} \mathrm{~d} z H^{(2)}(z)\left[\left(S_{\alpha}(\tilde{x} P)-P_{\alpha}(\tilde{x} S)\right) \mathrm{e}^{\mathrm{i} \kappa z(\tilde{x} P)}+\tilde{x}_{\alpha}(\tilde{x} S) M^{2} \int_{0}^{1} \mathrm{~d} \lambda \mathrm{i} \kappa z \lambda^{2} \mathrm{e}^{\mathrm{i} \kappa \lambda z(\tilde{x} P)}\right], \\
& =\frac{2}{M} \sum_{n=0}^{\infty} \frac{(\mathrm{i} \kappa(\tilde{x} P))^{n}}{n !} H_{n}^{(2)}\left\{S_{\alpha}(\tilde{x} P)-P_{\alpha}(\tilde{x} S)+\frac{n}{n+2} \tilde{x}_{\alpha} \frac{\tilde{x} S}{\tilde{x} P} M^{2}\right\} \\
\left\langle P S\left|M_{5 \alpha}^{\mathrm{tw} 3}\left(\kappa_{1} \tilde{x}, \kappa_{2} \tilde{x}\right)\right| P S\right\rangle & =-2 \int_{0}^{1} \mathrm{~d} \lambda \int_{0}^{1} \mathrm{~d} z H^{(3)}(z)\left[\tilde{x}_{\alpha}(\tilde{x} S) M \mathrm{i} \kappa z \lambda^{2}\right] \mathrm{e}^{\mathrm{i} \kappa \lambda z(\tilde{x} P)} \\
& =-2 \sum_{n=1}^{\infty} \frac{(\mathrm{i} \kappa(\tilde{x} P))^{n}}{n !} H_{n}^{(3)} \frac{n}{n+2} \tilde{x}_{\alpha} \frac{\tilde{x} S}{\tilde{x} P} M .
\end{aligned}
$$

The twist-2 part is in agreement with Jaffe and Ji's local expression (see Eq. (45) in 《]) and also with [19]. Adding up both local terms the matrix element of $M_{\alpha}\left(\kappa_{1} \tilde{x}, \kappa_{2} \tilde{x}\right)$ coincides with the corresponding one of 伆; its nonlocal version reads:

$$
\begin{aligned}
\left\langle P S\left|M_{5 \alpha}\left(\kappa_{1} \tilde{x}, \kappa_{2} \tilde{x}\right)\right| P S\right\rangle= & \frac{2}{M}\left(S_{\alpha}(\tilde{x} P)-P_{\alpha}(\tilde{x} S)\right) \int_{0}^{1} \mathrm{~d} z H^{(2)}(z) e_{0}(\mathrm{i} \zeta z) \\
& +\tilde{x}_{\alpha} \frac{\tilde{x} S}{\tilde{x} P} M \int_{0}^{1} \mathrm{~d} z\left(H^{(2)}(z)-H^{(3)}(z)\right)\left(e_{0}(\mathrm{i} \zeta z)+2 e_{2}(\mathrm{i} \zeta z)\right) .
\end{aligned}
$$

Obviously, the twist-2 distribution function $H^{(2)}(z)$ and the twist-3 distribution function $H^{(3)}(z)$ contribute destructive to the same $1 / Q^{2}$-behaviour of the cross section. A similar nonlocal twist-2 matrix element (modulo correction of the trace term) has been given in [20].

Now, we consider the more involved chiral-odd skew tensor operator. The matrix elements of the skew tensor operators of twist $\tau$ are obtained using the projectors determined by Eqs. (2.11) - (2.13):

$$
\begin{aligned}
&\left\langle P S\left|M_{5[\alpha \beta]}^{\mathrm{tw} 2}\left(\kappa_{1} \tilde{x}, \kappa_{2} \tilde{x}\right)\right| P S\right\rangle= \frac{2}{M} \int_{0}^{1} \mathrm{~d} \lambda \int_{0}^{1} \mathrm{~d} z H^{(2)}(z)\left[2 \lambda S_{[\alpha} P_{\beta]}(2+\mathrm{i} \kappa \lambda z(\tilde{x} P))\right. \\
&\left.+(1-\lambda) M^{2} \tilde{x}_{[\alpha}\left\{4(\mathrm{i} \kappa \lambda z) S_{\beta]}+(\mathrm{i} \kappa \lambda z)^{2}\left(S_{\beta]}(\tilde{x} P)+P_{\beta]}(\tilde{x} S)\right)\right\}\right] \mathrm{e}^{\mathrm{i} \kappa \lambda z(\tilde{x} P)} \\
&=\frac{2}{M} \sum_{n=0}^{\infty} \frac{(\mathrm{i} \kappa(\tilde{x} P))^{n}}{n !} H_{n}^{(2)}\left\{2 S_{[\alpha} P_{\beta]}+\frac{4 n}{(n+2)(n+1)} \frac{M^{2}}{\tilde{x} P} \tilde{x}_{[\alpha} S_{\beta]}+\frac{n(n-1)}{(n+2)(n+1)} \frac{M^{2}}{\tilde{x} P} \tilde{x}_{[\alpha}\left(S_{\beta]}+P_{\beta]} \frac{\tilde{x} S}{\tilde{x} P}\right)\right\} \\
&\left\langle P S\left|M_{5[\alpha \beta]}^{\mathrm{tw} 3}\left(\kappa_{1} \tilde{x}, \kappa_{2} \tilde{x}\right)\right| P S\right\rangle= 2 \int_{0}^{1} \mathrm{~d} \lambda \frac{1-\lambda^{2}}{\lambda} \int_{0}^{1} \mathrm{~d} z \mathrm{i} \kappa \lambda z H^{(3)}(z) M \tilde{x}_{[\alpha}\left\{S_{\beta]}+\mathrm{i} \kappa \lambda z P_{\beta]}(\tilde{x} S)\right\} \mathrm{e}^{\mathrm{i} \kappa \lambda z(\tilde{x} P)} \\
&=-2 \sum_{n=1}^{\infty} \frac{(\mathrm{i} \kappa(\tilde{x} P))^{n}}{n !} H_{n}^{(3)} \frac{2}{n+2} \frac{M}{\tilde{x} P} \tilde{x}_{[\alpha}\left(S_{\beta]}+(n-1) P_{\beta]} \frac{\tilde{x} S}{\tilde{x} P}\right), \\
&\left\langle P S\left|M_{5[\alpha \beta]}^{\mathrm{tw} 4}\left(\kappa_{1} \tilde{x}, \kappa_{2} \tilde{x}\right)\right| P S\right\rangle=-2 \int_{0}^{1} \mathrm{~d} \lambda \frac{1-\lambda}{\lambda} \int_{0}^{1} \mathrm{~d} z(\mathrm{i} \lambda \kappa z)^{2} H^{(4)}(z) M \tilde{x}_{[\alpha}\left\{S_{\beta]}(\tilde{x} P)-P_{\beta]}(\tilde{x} S)\right\} \mathrm{e}^{\mathrm{i} \kappa \lambda z(\tilde{x} P)} \\
&=-2 \sum_{n=2}^{\infty} \frac{(\mathrm{i} \kappa(\tilde{x} P))^{n}}{n !} H_{n}^{(4)} \frac{n-1}{n+1} \frac{M}{\tilde{x} P} \tilde{x}_{[\alpha}\left(S_{\beta]}-P_{\beta]} \frac{\tilde{x} S}{\tilde{x} P}\right) .
\end{aligned}
$$

Again, the moments of distributions functions of twist $\tau=2,3$ and 4 begin with $n=0,1$ and 2 , respectively. In addition, we remark that only those terms of the operator (2.12) contribute to the twist-3 structure function which result from the trace terms of (2.11). Analogous to the vector case the forward matrix element of the 'true' twist3 part of (2.12) vanishes. In Eq. (3.31) only the twist-4 operator contributes which result from the trace terms of (2.11). Let us also mention that after multiplication of (3.31) with $\tilde{x}_{\alpha}$ (or $\tilde{x}_{\beta}$ ) the matrix element vanishes because the corresponding vector operator does not contain any twist-four contribution. This is a simple but important property which may be traced back to the fact that the corresponding Young pattern $[n+2]=(n, 1,1)$ does allow only $n$ symmetrizations; it is therefore characteristic for any twist- 4 skew tensor operator. 
The matrix element of the original skew tensor operator is obtained as

$$
\begin{aligned}
\left\langle P S\left|M_{5[\alpha \beta]}\left(\kappa_{1} \tilde{x}, \kappa_{2} \tilde{x}\right)\right| P S\right\rangle & =\frac{4}{M} S_{[\alpha} P_{\beta]} \int_{0}^{1} \mathrm{~d} z H^{(2)}(z) e_{0}(\mathrm{i} \zeta z) \\
& +2 \tilde{x}_{[\alpha} P_{\beta]} \frac{\tilde{x} S}{(\tilde{x} P)^{2}} M \int_{0}^{1} \mathrm{~d} z\left\{H^{(2)}(z)\left[e_{0}(\mathrm{i} \zeta z)+2 e_{1}(\mathrm{i} \zeta z)+6 e_{2}(\mathrm{i} \zeta z)\right]\right. \\
& \left.\quad-H^{(3)}(z)\left[1+2 e_{0}(\mathrm{i} \zeta z)+6 e_{2}(\mathrm{i} \zeta z)\right]+H^{(4)}(z)\left[1+e_{0}(\mathrm{i} \zeta z)-2 e_{1}(\mathrm{i} \zeta z)\right]\right\} \\
& +2 \tilde{x}_{[\alpha} S_{\beta]} \frac{M}{\tilde{x} P} \int_{0}^{1} \mathrm{~d} z\left\{H^{(2)}(z)\left[e_{0}(\mathrm{i} \zeta z)-2 e_{1}(\mathrm{i} \zeta z)-2 e_{2}(\mathrm{i} \zeta z)\right]\right. \\
& \left.+H^{(3)}(z)\left[1+2 e_{2}(\mathrm{i} \zeta z)\right]-H^{(4)}(z)\left[1+e_{0}(\mathrm{i} \zeta z)-2 e_{1}(\mathrm{i} \zeta z)\right]\right\} .
\end{aligned}
$$

This finishes the determination of the nine nontrivial distribution functions which result from the forward matrix elements of the nonlocal light-cone quark operators of definite twist.

\section{RELATIONS BETWEEN NEW AND CONVENTIONAL DISTRIBUTION FUNCTIONS}

Obviously, since these new distribution functions are related to true traceless operators they differ from the conventional ones [4] for twist $\tau \geq 3$ at least by the contributions from the trace terms. As far as the scalar LC-operators are concerned which definitely are of twist-2 the new and the old distributions functions coincide. However, for the vector and (skew) tensor operators also the contributions of dynamical twist-2 differ from those of geometric twist-2.

In order to be able to compare conventional and new structure functions, we rewrite the matrix elements (3.10), (3.19) and (3.33) by choosing

$$
\tilde{x}_{\alpha}=x_{\alpha}-\frac{P_{\alpha}}{M^{2}}\left((x P)-\sqrt{(x P)^{2}-x^{2} M^{2}}\right), \quad P_{\alpha}=p_{\alpha}+\frac{1}{2} \tilde{x}_{\alpha} \frac{M^{2}}{\tilde{x} P}, \quad S_{\alpha}=S_{\alpha}^{\perp}+p_{\alpha} \frac{\tilde{x} S}{\tilde{x} P}-\frac{1}{2} \tilde{x}_{\alpha} \frac{\tilde{x} S}{(\tilde{x} P)^{2}} M^{2},
$$

where $p_{\alpha}$ is a light-like vector $\left(p^{2}=0\right.$ and $\left.p \cdot \tilde{x}=P \cdot \tilde{x}\right)$ and $S_{\alpha}^{\perp}$ is the transversal spin-polarization. Using this parametrization we obtain

$$
\begin{aligned}
& \left\langle P S\left|O_{5 \alpha}\left(\kappa_{1} \tilde{x}, \kappa_{2} \tilde{x}\right)\right| P S\right\rangle=2 p_{\alpha} \frac{\tilde{x} S}{\tilde{x} P} \int_{0}^{1} \mathrm{~d} z G^{(2)}(z) e_{0}(\mathrm{i} \zeta z)+2 S_{\alpha}^{\perp} \int_{0}^{1} \mathrm{~d} z\left\{G^{(2)}(z) e_{1}(\mathrm{i} \zeta z)+G^{(3)}(z)\left[e_{0}(\mathrm{i} \zeta z)-e_{1}(\mathrm{i} \zeta z)\right]\right\} \\
& \quad-\tilde{x}_{\alpha} \frac{\tilde{x} S}{(\tilde{x} P)^{2}} M^{2} \int_{0}^{1} \mathrm{~d} z\left\{G^{(4)}(z)\left[e_{0}(\mathrm{i} \zeta z)-3 e_{1}(\mathrm{i} \zeta z)+2 \int_{0}^{1} \mathrm{~d} \lambda e_{1}(\mathrm{i} \zeta \lambda z)\right]-G^{(2)}(z)\left[e_{1}(\mathrm{i} \zeta z)-2 \int_{0}^{1} \mathrm{~d} \lambda e_{1}(\mathrm{i} \zeta \lambda z)\right]\right. \\
& \left.+4 G^{(3)}(z)\left[e_{1}(\mathrm{i} \zeta z)-\int_{0}^{1} \mathrm{~d} \lambda e_{1}(\mathrm{i} \zeta \lambda z)\right]\right\} \\
& \left\langle P\left|O_{\alpha}\left(\kappa_{1} \tilde{x}, \kappa_{2} \tilde{x}\right)\right| P\right\rangle=2 p_{\alpha} \int_{0}^{1} \mathrm{~d} z F^{(2)}(z) e_{0}(\mathrm{i} \zeta z)+\tilde{x}_{\alpha} \frac{M^{2}}{\tilde{x} P} \int_{0}^{1} \mathrm{~d} z\left\{F^{(4)}(z) e_{0}(\mathrm{i} \zeta z)+\left[F^{(2)}(z)-F^{(4)}(z)\right] e_{1}(\mathrm{i} \zeta z)\right\} \\
& \left\langle P\left|\bar{\psi}\left(\kappa_{1} \tilde{x}\right) U\left(\kappa_{1} \tilde{x}, \kappa_{2} \tilde{x}\right) \psi\left(\kappa_{2} \tilde{x}\right)\right| P\right\rangle=2 \int_{0}^{1} \mathrm{~d} z E^{(3)}(z) e_{0}(\mathrm{i} \zeta z) \\
& \left\langle P S\left|M_{5[\alpha \beta]}\left(\kappa_{1} \tilde{x}, \kappa_{2} \tilde{x}\right)\right| P S\right\rangle=\frac{4}{M} S_{[\alpha}^{\perp} p_{\beta]} \int_{0}^{1} \mathrm{~d} z H^{(2)}(z) e_{0}(\mathrm{i} \zeta z) \\
& \quad+2 \tilde{x}_{[\alpha} p_{\beta]} \frac{\tilde{x} S}{(\tilde{x} P)^{2}} M \int_{0}^{1} \mathrm{~d} z\left\{4 H^{(2)}(z) e_{2}(\mathrm{i} \zeta z)-2 H^{(3)}(z)\left[e_{0}(\mathrm{i} \zeta z)+2 e_{2}(\mathrm{i} \zeta z)\right]\right\} \\
& \quad-2 \tilde{x}_{[\alpha} S_{\beta]}^{\perp} \frac{M}{\tilde{x} P} \int_{0}^{1} \mathrm{~d} z\left\{2 H^{(2)}(z)\left[e_{1}(\mathrm{i} \zeta z)+e_{2}(\mathrm{i} \zeta z)\right]-H^{(3)}(z)\left[1+2 e_{2}(\mathrm{i} \zeta z)\right]+H^{(4)}(z)\left[1+e_{0}(\mathrm{i} \zeta z)-2 e_{1}(\mathrm{i} \zeta z)\right]\right\}
\end{aligned}
$$

Let us note that after multiplication of (4.5) with $\tilde{x}_{\alpha}\left(\right.$ or $\left.\tilde{x}_{\beta}\right)$ the last part of the matrix element vanishes $\left(\tilde{x} \cdot S^{\perp}=0\right)$. This means that the dynamical twist-4 function $h_{3}(z)$ can be ignored in the case of the vector operator $M_{5 \alpha}\left(\kappa_{1} \tilde{x}, \kappa_{2} \tilde{x}\right)$ but not in the case of the more general tensor operator $M_{5[\alpha \beta]}\left(\kappa_{1} \tilde{x}, \kappa_{2} \tilde{x}\right)$. 


\begin{tabular}{c|ccc|}
\hline \hline Twist $t$ & 2 & 3 & 4 \\
& $O(1)$ & $O(1 / Q)$ & $O\left(1 / Q^{2}\right)$ \\
\hline spin ave. & $f_{1}=F^{(2)}$ & $\underline{e}=E^{(3)}$ & $f_{4}=F^{(4)} / 2+F_{f}\left(F^{(2)}, F^{(4)}\right) / 2$ \\
$S_{\|}$ & $g_{1}=G^{(2)}$ & $\underline{h_{L}}=H^{(3)}+F_{L}\left(H^{(2)}, H^{(3)}\right)$ & $g_{3}=-G^{(4)} / 2+F_{g}\left(G^{(2)}, G^{(3)}, G^{(4)}\right) / 2$ \\
$S_{\perp}$ & $\underline{h_{1}}=H^{(2)}$ & $g_{T}=G^{(3)}+F_{T}\left(G^{(2)}, G^{(3)}\right)$ & $\underline{h_{3}}=H^{(4)} / 2+F_{h}\left(H^{(2)}, H^{(3)}, H^{(4)}\right) / 2$ \\
\hline \hline
\end{tabular}

TABLE I. Spin, dynamical twist and chiral classification of the nucleon structure functions.

On the other hand, these matrix elements in terms of the nine parton distributions introduced by Jaffe and Ji [4] are given as follows:

$$
\begin{aligned}
& \left\langle P S\left|O_{5 \alpha}\left(\kappa_{1} \tilde{x}, \kappa_{2} \tilde{x}\right)\right| P S\right\rangle=2\left[p_{\alpha} \frac{\tilde{x} S}{\tilde{x} P} \int_{0}^{1} \mathrm{~d} z \mathrm{e}^{\mathrm{i} \zeta z} g_{1}(z)+S_{\alpha}^{\perp} \int_{0}^{1} \mathrm{~d} z \mathrm{e}^{\mathrm{i} \zeta z} g_{T}(z)+\tilde{x}_{\alpha} \frac{\tilde{x} S}{(\tilde{x} P)^{2}} M^{2} \int_{0}^{1} \mathrm{~d} z \mathrm{e}^{\mathrm{i} \zeta z} g_{3}(z)\right] \\
& \left\langle P\left|O_{\alpha}\left(\kappa_{1} \tilde{x}, \kappa_{2} \tilde{x}\right)\right| P\right\rangle=2\left[p_{\alpha} \int_{0}^{1} \mathrm{~d} z \mathrm{e}^{\mathrm{i} \zeta z} f_{1}(z)+\tilde{x}_{\alpha} \frac{M^{2}}{\tilde{x} P} \int_{0}^{1} \mathrm{~d} z \mathrm{e}^{\mathrm{i} \zeta z} f_{4}(z)\right] \\
& \left\langle P\left|\bar{\psi}\left(\kappa_{1} \tilde{x}\right) U\left(\kappa_{1} \tilde{x}, \kappa_{2} \tilde{x}\right) \psi\left(\kappa_{2} \tilde{x}\right)\right| P\right\rangle=2 M \int_{0}^{1} \mathrm{~d} z \mathrm{e}^{\mathrm{i} \zeta z} e(z), \\
& \left\langle P S\left|M_{5[\alpha \beta]}\left(\kappa_{1} \tilde{x}, \kappa_{2} \tilde{x}\right)\right| P S\right\rangle=\frac{4}{M}\left[S_{[\alpha}^{\perp} p_{\beta]} \int_{0}^{1} \mathrm{~d} z \mathrm{e}^{\mathrm{i} \zeta z} h_{1}(z)-\tilde{x}_{[\alpha} p_{\beta]} \frac{\tilde{x} S M^{2}}{(\tilde{x} P)^{2}} \int_{0}^{1} \mathrm{~d} z \mathrm{e}^{\mathrm{i} \zeta z} h_{L}(z)-\tilde{x}_{[\alpha} S_{\beta]}^{\perp} \frac{M^{2}}{\tilde{x} P} \int_{0}^{1} \mathrm{~d} z \mathrm{e}^{\mathrm{i} \zeta z} h_{3}(z)\right] .
\end{aligned}
$$

From the expressions (4.2) - 4.5) and (4.6) - 4.9) it is obvious that the conventional structure functions of twist $t \geq 3$ contain contributions also of lower geometric twist. In Tab. I we classify them according to their spin, dynamical twist and chirality and we also note their content of geometric twist: The parton distributions in the first row are spin-independent, those in the second and third row describe longitudinally $\left(S_{\|}\right)$and transversely $\left(S_{\perp}\right)$ polarized nucleons, respectively. The underlined distributions are referred to as chiral-odd, because they correspond to chirality-violating Dirac matrix structures $\Gamma=\left\{\sigma_{\alpha \beta} \mathrm{i} \gamma_{5}, 1\right\}$. The other distributions are termed chiral-even, because of the chirality-conserving structures $\Gamma=\left\{\gamma_{\alpha}, \gamma_{\alpha} \gamma_{5}\right\}$.

Re-expressing the truncated exponentials and performing appropriate variable transformations we obtain the following relations, which give the interrelation between the structure functions of different twist:

$$
\begin{aligned}
g_{1}(z) & =G^{(2)}(z) \\
g_{T}(z) & =G^{(3)}(z)+\int_{z}^{1} \frac{\mathrm{d} y}{y}\left(G^{(2)}-G^{(3)}\right)(y) \\
2 g_{3}(z) & =-G^{(4)}(z)+\int_{z}^{1} \frac{\mathrm{d} y}{y}\left\{\left(G^{(2)}-4 G^{(3)}+3 G^{(4)}\right)(y)+2 \ln \left(\frac{z}{y}\right)\left(G^{(2)}-2 G^{(3)}+G^{(4)}\right)(y)\right\}, \\
f_{1}(z) & =F^{(2)}(z) \\
2 f_{4}(z) & =F^{(4)}(z)+\int_{z}^{1} \frac{\mathrm{d} y}{y}\left(F^{(2)}-F^{(4)}\right)(y) \\
e(z) & =E^{(3)}(z) \\
h_{1}(z) & =H^{(2)}(z) \\
h_{L}(z) & =H^{(3)}(z)+2 z \int_{z}^{1} \frac{\mathrm{d} y}{y^{2}}\left(H^{(2)}-H^{(3)}\right)(y), \\
2 h_{3}(z) & =H^{(4)}(z)+\int_{z}^{1} \frac{\mathrm{d} y}{y}\left\{2\left(H^{(2)}-H^{(4)}\right)(y)-2 \frac{z}{y}\left(H^{(2)}-H^{(3)}\right)(y)-\delta\left(\frac{z}{y}\right)\left(H^{(3)}-H^{(4)}\right)(y)\right\} .
\end{aligned}
$$

These relations between the conventional and the new quark distribution functions hold for $1 \geq z \geq 0$; the corresponding antiquark distribution functions are obtained for $z \rightarrow-z$. Furthermore, we observe that both decompositions coincide in the leading terms, but differ at higher order. For instance, $g_{1}(z)$ and $h_{1}(z)$ are genuine geometric twist-2 
structure functions and $e(z)$ is a genuine twist-3 function. Moreover, the parton distribution functions $g_{T}(z), f_{1}(z)$ and $h_{L}(z)$ with dynamical twist $t=3$ also contain contributions of geometrical twist $\tau=2$ and 3 . Additionally, the dynamical twist $t=4$ functions $g_{3}(z)$ and $h_{3}(z)$ contain geometrical twist-2, twist-3 as well as twist- 4 parts and the dynamical twist $t=4$ function $f_{4}(z)$ contains geometrical twist-2 and twist- 4 .

These relationship may be inverted. The nontrivial inverse relations are:

$$
\begin{aligned}
& G^{(3)}(z)=g_{T}(z)+\frac{1}{z} \int_{z}^{1} \mathrm{~d} y\left(g_{T}-g_{1}\right)(y) \\
& G^{(4)}(z)=-\left\{2 g_{3}(z)+\frac{1}{z^{2}} \int_{z}^{1} \mathrm{~d} y y\left(6 g_{3}+4 g_{T}-g_{1}\right)(y)-\frac{1}{z^{2}} \int_{z}^{1} \mathrm{~d} y y \ln \left(\frac{z}{y}\right)\left(2 g_{3}+4 g_{T}-3 g_{1}\right)(y)\right\} \\
& F^{(4)}(z)=2 f_{4}(z)+\frac{1}{z} \int_{z}^{1} \mathrm{~d} y\left(2 f_{4}-f_{1}\right)(y) \\
& H^{(3)}(z)=h_{L}(z)+\frac{2}{z} \int_{z}^{1} \mathrm{~d} y\left(h_{L}-h_{1}\right)(y) \\
& H^{(4)}(z)=2\left\{h_{3}(z)+\frac{1}{z^{2}} \int_{z}^{1} \mathrm{~d} y y\left(2 h_{3}-h_{L}\right)(y)+\frac{1}{z^{2}} \int_{z}^{1} \mathrm{~d} y y \ln \left(\frac{z}{y}\right)\left(h_{L}-h_{1}\right)(y)\right\} .
\end{aligned}
$$

The relation between the moments may be read off from Eqs. (4.2) - (4.5) as follows:

$$
\begin{aligned}
g_{1 n} & =G_{n}^{(2)}, \\
g_{T n} & =G_{n}^{(3)}+\frac{1}{n+1}\left(G_{n}^{(2)}-G_{n}^{(3)}\right), \\
2 g_{3 n} & =-G_{n}^{(4)}+\frac{1}{n+1}\left(G_{n}^{(2)}-4 G_{n}^{(3)}+3 G_{n}^{(4)}\right)-\frac{2}{(n+1)^{2}}\left(G_{n}^{(2)}-2 G_{n}^{(3)}+G_{n}^{(4)}\right), \\
f_{1 n} & =F_{n}^{(2)}, \\
2 f_{4 n} & =F_{n}^{(4)}+\frac{1}{n+1}\left(F_{n}^{(2)}-F_{n}^{(4)}\right), \\
e_{n} & =E_{n}^{(3)}, \\
h_{1 n} & =H_{n}^{(2)}, \\
h_{L n} & =H_{n}^{(3)}+\frac{2}{n+2}\left(H_{n}^{(2)}-H_{n}^{(3)}\right), \\
2 h_{3 n} & =H_{n}^{(4)}+\frac{2}{n+1}\left(H_{n}^{(2)}-H_{n}^{(4)}\right)-\frac{2}{n+2}\left(H_{n}^{(2)}-H_{n}^{(3)}\right)-\delta_{n 0}\left(H_{n}^{(3)}-H_{n}^{(4)}\right) .
\end{aligned}
$$

In terms of the moments the relations between conventional and new distribution functions may be easily inverted. The nontrivial inverse relations are:

$$
\begin{array}{llr}
G_{n}^{(3)}=g_{T n}+\frac{1}{n}\left(g_{T n}-g_{1 n}\right), & n>0 \\
G_{n}^{(4)}=-\left\{2 g_{3 n}+\frac{1}{n-1}\left(6 g_{3 n}+4 g_{T n}-g_{1 n}\right)+\frac{1}{n(n-1)}\left(2 g_{3 n}+4 g_{T n}-3 g_{1 n}\right)\right\}, & n>1 \\
F_{n}^{(4)}=2 f_{4 n}+\frac{1}{n}\left(2 f_{4 n}-f_{1 n}\right), & n>0 \\
H_{n}^{(3)}=h_{L n}+\frac{2}{n}\left(h_{L n}-h_{1 n}\right), & n>0 \\
H_{n}^{(4)}=2\left\{h_{3 n}+\frac{1}{n-1}\left(2 h_{3 n}-h_{L n}\right)-\frac{1}{n(n-1)}\left(h_{L n}-h_{1 n}\right)\right\}, & n>1 .
\end{array}
$$

The nontrivial relationships between the conventional and the new distribution functions are much simpler than to be assumed by a first glance at the expressions like (3.10), (3.19) and (3.33). They show that the conventional distribution functions are determined by the new ones of the same as well as lower geometrical twist, and vice versa (with respect to dynamical twist). Obviously, the same holds for their moments. In principle, this allows to determine, e.g., the new distribution amplitudes from the experimental data if these are known for the conventional ones. At least, this should be possible for the lowest moments. 


\section{WANDZURA-WILCZEK-LIKE RELATIONS}

As an immediate consequence of the interrelations $(4.10)-(4.18)$ and $(4.19)-(4.23)$ between the conventional structure functions and the new ones of genuine geometric twist we are able to derive the decomposition of the conventional structure functions into its parts of genuine twist. Thereby, we also obtain new Wandzura-Wilczek-like relations for the conventional structure functions and also new sum rules of the type of the Burkhardt-Cottingham sum rule.

Let us introduce the usual notation $g_{T}(z)=g_{1}(z)+g_{2}(z)$ and the following decomposition of $g_{2}(z)=g_{2}^{(2)}(z)+g_{2}^{(3)}(z)$ into its parts $g_{2}^{(2)}(z)$ and $g_{2}^{(3)}(z)$ of genuine twist-2 and twist-3, respectively, part of $g_{2}(z)$. Then, substituting (4.10) into (4.11), we get

$$
\begin{aligned}
& g_{2}^{(2)}(z)=-g_{1}(z)+\int_{z}^{1} \frac{\mathrm{d} y}{y} g_{1}(y) \\
& g_{2}^{(3)}(z)=g_{2}(z)+g_{1}(z)-\int_{z}^{1} \frac{\mathrm{d} y}{y} g_{1}(y),
\end{aligned}
$$

where (5.1) is just the Wandzura-Wilczek relation for the twist-2 part [21]. On the other hand, the analogous relation (5.2) for the twist-3 part, which is an immediate consequence of the above definition, has already been obtained in the framework of the local OPE [22] and the nonlocal OPE 23]. For the moments we obtain

$$
\begin{aligned}
& g_{2 n}^{(2)}=-g_{1 n}+\frac{1}{n+1} g_{1 n}, \\
& g_{2 n}^{(3)}=g_{2 n}+\frac{n}{n+1} g_{1 n}, \quad n>0 .
\end{aligned}
$$

Obviously, from the relation (5.3) for $n=0$ the Burkhardt- Cottingham sum rule 24] follows:

$$
\int_{0}^{1} \mathrm{~d} z g_{2}(z)=0 \text {. }
$$

Of course, these relationships are well known. However, with the help of the results of the preceeding chapter we are able to generalize these relationships also to the other nontrivial structure functions. Using the formulas (4.10), (4.11) (4.12) and (4.19), we obtain Wandzura-Wilczek-like integral relations for geometric twist parts of the function $g_{3}(z)=g_{3}^{(2)}(z)+g_{3}^{(3)}(z)+g_{3}^{(4)}(z)$ as follows:

$$
\begin{aligned}
g_{3}^{(2)}(z)= & \int_{z}^{1} \frac{\mathrm{d} y}{y}\left\{\frac{g_{1}(y)}{2}+\ln \left(\frac{z}{y}\right) g_{1}(y)\right\} \\
g_{3}^{(3)}(z)= & -2 \int_{z}^{1} \frac{\mathrm{d} y}{y}\left(g_{1}+g_{2}\right)(y)-2 \int_{z}^{1} \frac{\mathrm{d} y}{y^{2}} \int_{y}^{1} \mathrm{~d} u g_{2}(u) \\
& -2 \int_{z}^{1} \frac{\mathrm{d} y}{y} \ln \left(\frac{z}{y}\right)\left(g_{1}+g_{2}\right)(y)-2 \int_{z}^{1} \frac{\mathrm{d} y}{y^{2}} \ln \left(\frac{z}{y}\right) \int_{y}^{1} \mathrm{~d} u g_{2}(u), \\
g_{3}^{(4)}(z)= & g_{3}(z)+\frac{1}{2} \int_{z}^{1} \frac{\mathrm{d} y}{y}\left(3 g_{1}+4 g_{2}\right)(y)+2 \int_{z}^{1} \frac{\mathrm{d} y}{y^{2}} \int_{y}^{1} \mathrm{~d} u g_{2}(u) \\
& +\int_{z}^{1} \frac{\mathrm{d} y}{y} \ln \left(\frac{z}{y}\right)\left(g_{1}+2 g_{2}\right)(y)+2 \int_{z}^{1} \frac{\mathrm{d} y}{y^{2}} \ln \left(\frac{z}{y}\right) \int_{y}^{1} \mathrm{~d} u g_{2}(u) .
\end{aligned}
$$

Due to the fact that $g_{3}(z)$ contains twist-2, twist-3 as well as twist- 4 we have obtained three integral relations. For example, Eq. (5.6) demonstrates that the twist-2 part $g_{3}^{(2)}(z)$ can be expressed in terms of the twist-2 function $g_{1}(z)$. The relations for the moments are:

$$
\begin{aligned}
& g_{3 n}^{(2)}=\frac{1}{2(n+1)} g_{1 n}-\frac{1}{(n+1)^{2}} g_{1 n}, \\
& g_{3 n}^{(3)}=-\frac{2}{n+1}\left(g_{1 n}+g_{2 n}\right)-\frac{2}{(n+1) n} g_{2 n}+\frac{2}{(n+1)^{2}}\left(g_{1 n}+g_{2 n}\right)+\frac{2}{(n+1)^{2} n} g_{2 n}, \quad n>0 \\
& g_{3 n}^{(4)}=g_{3 n}+\frac{1}{2(n+1)}\left(3 g_{1 n}+4 g_{2 n}\right)+\frac{2}{(n+1) n} g_{2 n}-\frac{1}{(n+1)^{2}}\left(g_{1 n}+2 g_{2 n}\right)-\frac{2}{(n+1)^{2} n} g_{2 n}, \quad n>1 .
\end{aligned}
$$


For $n=0,1$ in $(5.9)$ we find the sum rules

$$
\int_{0}^{1} \mathrm{~d} z g_{3}(z)=-\frac{1}{2} \int_{0}^{1} \mathrm{~d} z g_{1}(z), \quad \int_{0}^{1} \mathrm{~d} z z g_{3}^{(2)}(z)=0
$$

as well as

$$
\int_{0}^{1} \mathrm{~d} z z g_{3}^{(3)}(z)=-\frac{1}{2} \int \mathrm{d} z z\left(g_{1}+2 g_{2}\right)(z)
$$

Substituting (4.13) into (4.14), we get the integral relations for the twist-2 and twist-4 part of $f_{4}(z)=f_{4}^{(2)}(z)+$ $f_{4}^{(4)}(z)$

$$
\begin{aligned}
& f_{4}^{(2)}(z)=\frac{1}{2} \int_{z}^{1} \frac{\mathrm{d} y}{y} f_{1}(y) \\
& f_{4}^{(4)}(z)=f_{4}(z)-\frac{1}{2} \int_{z}^{1} \frac{\mathrm{d} y}{y} f_{1}(y) .
\end{aligned}
$$

The corresponding relations for the moments read

$$
\begin{aligned}
f_{4 n}^{(2)} & =\frac{1}{2(n+1)} f_{1 n}, \\
f_{4 n}^{(4)} & =f_{4 n}-\frac{1}{2(n+1)} f_{1 n}, \quad n>0 .
\end{aligned}
$$

If we now substitute (4.16) into (4.17) and using $h_{L}(z)=h_{2}(z)+h_{1}(z) / 2$ and $h_{2}(z)=h_{2}^{(2)}(z)+h_{2}^{(3)}(z)$, we derive

$$
\begin{aligned}
& h_{2}^{(2)}(z)=-\frac{h_{1}(z)}{2}+2 z \int_{z}^{1} \frac{\mathrm{d} y}{y^{2}} h_{1}(y) \\
& h_{2}^{(3)}(z)=h_{2}(z)+\frac{h_{1}(z)}{2}-2 z \int_{z}^{1} \frac{\mathrm{d} y}{y^{2}} h_{1}(y),
\end{aligned}
$$

where (5.18) is a twist-2 Wandzura-Wilczek-like relation which was obtained earlier by Jaffe and Ji [- 4 (see also [20]). Obviously, (5.19) is the corresponding twist-3 relation. For the moments we obtain

$$
\begin{aligned}
& h_{2 n}^{(2)}=-\frac{h_{1 n}}{2}+\frac{2}{n+2} h_{1 n}, \\
& h_{2 n}^{(3)}=h_{2 n}+\frac{h_{1 n}}{2}-\frac{2}{n+2} h_{1 n}, \quad n>0 .
\end{aligned}
$$

For $n=0,1,2$ in 5.20 we observe the following sum rules

$$
\int_{0}^{1} \mathrm{~d} z h_{2}(z)=\frac{1}{2} \int_{0}^{1} \mathrm{~d} z h_{1}(z), \quad \int_{0}^{1} \mathrm{~d} z z h_{2}^{(2)}(z)=\frac{1}{6} \int_{0}^{1} \mathrm{~d} z z h_{2}(z), \quad \int_{0}^{1} \mathrm{~d} z z^{2} h_{2}^{(2)}(z)=0 .
$$

Furthermore, using the formulas $(4.16), 4.17)$ (4.18) and (4.22), we obtain the integral relations for the structure function $h_{3}(z)=h_{3}^{(2)}(z)+h_{3}^{(3)}(z)+h_{3}^{(4)}(z)$ as follows:

$$
\begin{aligned}
& h_{3}^{(2)}(z)=\int_{z}^{1} \frac{\mathrm{d} y}{y} h_{1}(y)-z \int_{z}^{1} \frac{\mathrm{d} y}{y^{2}} h_{1}(y) \\
& h_{3}^{(3)}(z)=z \int_{z}^{1} \frac{\mathrm{d} y}{y^{2}}\left(h_{2}+\frac{h_{1}}{2}\right)(y)+2 z \int_{z}^{1} \frac{\mathrm{d} y}{y^{3}} \int_{y}^{1} \mathrm{~d} u\left(h_{2}-\frac{h_{1}}{2}\right)(u) \\
& h_{3}^{(4)}(z)=h_{3}(z)-\int_{z}^{1} \frac{\mathrm{d} y}{y} h_{1}(y)-z \int_{z}^{1} \frac{\mathrm{d} y}{y^{2}}\left(h_{2}-\frac{h_{1}}{2}\right)(y)-2 z \int_{z}^{1} \frac{\mathrm{d} y}{y^{3}} \int_{y}^{1} \mathrm{~d} u\left(h_{2}-\frac{h_{1}}{2}\right)(u),
\end{aligned}
$$


and the relations for the corresponding moments

$$
\begin{aligned}
& h_{3 n}^{(2)}=\frac{1}{n+1} h_{1 n}-\frac{1}{n+2} h_{1 n}, \\
& h_{3 n}^{(3)}=\frac{1}{n+2}\left(h_{2 n}+\frac{h_{1 n}}{2}\right)+\frac{2}{(n+2) n}\left(h_{2 n}-\frac{h_{1 n}}{2}\right), \quad n>0 \\
& h_{3 n}^{(4)}=h_{3 n}-\frac{1}{n+1} h_{1 n}-\frac{1}{n+2}\left(h_{2 n}-\frac{h_{1 n}}{2}\right)-\frac{2}{(n+2) n}\left(h_{2 n}-\frac{h_{1 n}}{2}\right), \quad n>1 .
\end{aligned}
$$

Also here we obtain some useful sum rule from (5.26) in the case $n=0$, namely,

$$
\int_{0}^{1} \mathrm{~d} z h_{3}(z)=\frac{1}{2} \int_{0}^{1} \mathrm{~d} z h_{1}(z)
$$

Let us note that Jaffe and Ji ignored in their investigations [4] the function $h_{3}(z)$ because they claimed that it is twist- 4 and can be neglected. However, this assumption is only true in the case of the vector operator $M_{5 \alpha}\left(\kappa_{1} \tilde{x}, \kappa_{2} \tilde{x}\right)$ but not in the case of the tensor operator $M_{5[\alpha \beta]}\left(\kappa_{1} \tilde{x}, \kappa_{2} \tilde{x}\right)$. As we have seen in Eq. (4.18), then the function $h_{3}(z)$

contains twist-2, twist-3 as well as twist- 4 parts. Moreover, the twist-2 part $h_{3}^{(2)}(z)$ is given in terms of the twist-2 function $h_{1}(z)$ in (5.23). Therefore, the structure function $h_{3}(z)$ is experimentally relevant also at the twist- 2 and twist-3 level and cannot be ignored in these cases.

\section{CONCLUSIONS}

Using the notion of geometric twist, we discussed the calculation of the forward matrix elements for those nonlocal LC-operators which correspond to the independent tensor structures in polarized nucleon matrix elements of the type $\left\langle P S\left|\bar{\psi}\left(\kappa_{1} \tilde{x}\right) \Gamma U\left(\kappa_{1} \tilde{x}, \kappa_{2} \tilde{x}\right) \psi\left(\kappa_{2} \tilde{x}\right)\right| P S\right\rangle$. We have found nine independent forward distribution functions with well defined twist $\tau$ which, for twist $\tau \geq 3$, differ from the conventional ones. From the field theoretical point of view this Lorentz invariant classification is the most appropriate frame of introducing distribution functions since the separation of different (geometric) twist is unique and independent from the special kinematics of the process. Only the operators of definite geometric twist will have the correct mixing behaviour under renormalization. A very useful property of the nonlocal (and local) LC operators of definite twist $\tau$ is that they are obtained from the original, i.e., undecomposed LC-operators by the application of corresponding projection operators. An essential result of our calculations is the relation between the new parton distribution functions and those given by Jaffe and Ji [4]. In addition we have also given the moments of all the distribution functions. These relations demonstrate the interrelations between the different twist definitions. One principal result of this paper are Wandzura-Wilczek-like relations between the dynamical twist distributions which we have obtained by means of our interrelation rules in natural manner. An advantage in our approach was that we used operators with geometric twist which allowed us to reveal the WandzuraWilczek-like relations between the dynamical twist distribution functions. The same procedure may be applied to the twist decomposition of the meson wave functions [25,26. Finally, in Appendix A, we have also written the nonlocal (and local) LC-operators by using the inner derivatives on the light-cone.

\section{ACKNOWLEDGMENTS}

The authors are grateful to J. Blümlein, D. Robaschik and S. Neumeier for useful discussions. They also grateful acknowledge stimulating discussions during the Leiden workshop on Polarized and Unpolarized Deep Inelastic LeptonHadron Scattering. M.L. acknowledges P. Ball and A.V. Radyushkin for stimulating discussions, furthermore to V.K. Dobrev for correspondence. Additionally, M.L. would like to express his gratitude to the Graduate College "Quantum field theory" at Center for Theoretical Sciences of Leipzig University for financial support. We would like to thank the anonymous referee for some helpful comments.

\section{APPENDIX A: TWIST OPERATORS AND THE INTERIOR DERIVATIVE ON THE LIGHT-CONE}

In this Appendix we like to rewrite the $\mathrm{LC}-$ operators of definite twist $\tau$ and, in addition, we formulate the property of tracelessness of such LC-operators by using internal derivatives on the light-cone. 
Let us now introduce the notion of interior differential operators on the light-cone $K_{4}=\left\{\tilde{x} \in \mathbb{M}^{4}, \tilde{x}^{2}=0\right\}$ which, for the first time, has been used in order to characterize (irreducible) symmetric tensor representations of $S O(4)$ on the cone and their graded algebra $P\left(K_{4}^{n}\right)=\oplus_{n=0}^{\infty} K_{4}^{n}$, where $K_{4}^{n}$ is the space of homogeneous polynomials $T_{n}(\tilde{x})$ of degree $n$ on the cone [27,28]:

A differential operator $Q$ is said to be an interior differential operator iff

$$
\left.Q\left(x^{2} T_{n}(x)\right)\right|_{x^{2} \equiv \tilde{x}^{2}=0}=0 .
$$

For example, the generators of dilation $X=1+\tilde{x} \tilde{\partial}$ and rotations $X_{\mu \nu}=\tilde{x}_{\nu} \tilde{\partial}_{\mu}-\tilde{x}_{\mu} \tilde{\partial}_{\nu}$ are first order interior differential operators on the light-cone. Obviously, they leave the space $K_{4}^{n}$ invariant.

A further interior differential operator $\mathrm{d}_{\mu}$ of second order may be introduced by the following requirements:

(a) it should be a lowering operator, i.e. mapping a homogeneous polynomial of degree $n$ (of $\tilde{x}$ ) into a homogeneous polynomial of degree $n-1, K_{4}^{n} \rightarrow K_{4}^{n-1}$;

(b) it should behave as a vector under rotations,

$$
\left[X_{\mu \nu}, \mathrm{d}_{\lambda}\right]=\delta_{\mu \lambda} \mathrm{d}_{\nu}-\delta_{\nu \lambda} \mathrm{d}_{\mu}
$$

(c) it should be the lowest order differential operator satisfying (a) and (b).

Choosing the normalization of this interior derivative so that $2 \mathrm{~d}_{\mu}$ is the generator of special conformal transformations of massless 0 -helicity representations of the conformal Lie algebra $\mathfrak{s o}(4,2)$, the interior derivative is given as

$$
\left.\mathrm{d}_{\alpha} f(\tilde{x}) \equiv\left\{(1+x \partial) \partial_{\alpha}-\frac{1}{2} x_{\alpha} \square\right\} f(x)\right|_{x=\tilde{x}},
$$

with the following properties

$$
\mathrm{d}^{2}=0, \quad\left[\mathrm{~d}_{\alpha}, \mathrm{d}_{\beta}\right]=0 \quad \text { and } \quad \mathrm{d}_{\alpha} \tilde{x}^{2}=\tilde{x}^{2}\left(\mathrm{~d}_{\alpha}+2 \tilde{\partial}_{\alpha}\right) .
$$

Obviously, $\mathrm{d}_{\mu}$ is a second order interior differential operator which together with $\tilde{x}_{\mu}, X$ and $X_{\mu \nu}$ satisfies the conformal Lie algebra $\mathfrak{s o}(4,2)$ in $\tilde{x}$-space; especially there holds the commutator relation

$$
\left[\mathrm{d}_{\mu}, \tilde{x}_{\nu}\right]=\delta_{\mu \nu} X+X_{\mu \nu} .
$$

In that terminology $\tilde{x}$ is a raising operator which plays the role of "momentum" in the conformal algebra:

$$
\left[\tilde{x}_{\mu}, \tilde{x}_{\nu}\right]=0, \quad \tilde{x}^{2}=0 .
$$

Now, we rewrite the nonlocal LC-operators (2.8) - (2.15) with the help of the above interior derivatives:

$$
\begin{aligned}
O_{\alpha}^{\mathrm{tw} 2}\left(\kappa_{1} \tilde{x}, \kappa_{2} \tilde{x}\right)= & -\int_{0}^{1} \mathrm{~d} \lambda(\ln \lambda) \mathrm{d}_{\alpha} \tilde{x}^{\mu} O_{\mu}\left(\kappa_{1} \lambda \tilde{x}, \kappa_{2} \lambda \tilde{x}\right) \\
O_{\alpha}^{\mathrm{tw} 3}\left(\kappa_{1} \tilde{x}, \kappa_{2} \tilde{x}\right)= & -\int_{0}^{1} \mathrm{~d} \lambda(\ln \lambda)\left\{X^{2} \delta_{\alpha}^{\mu}-\mathrm{d}_{\alpha} \tilde{x}^{\mu}-\tilde{x}_{\alpha} \mathrm{d}^{\mu}\right\} O_{\mu}\left(\kappa_{1} \lambda \tilde{x}, \kappa_{2} \lambda \tilde{x}\right) \\
O_{\alpha}^{\mathrm{tw} 4}\left(\kappa_{1} \tilde{x}, \kappa_{2} \tilde{x}\right)= & -\int_{0}^{1} \mathrm{~d} \lambda(\ln \lambda) \tilde{x}_{\alpha} \mathrm{d}^{\mu} O_{\mu}\left(\kappa_{1} \lambda \tilde{x}, \kappa_{2} \lambda \tilde{x}\right) \\
M_{[\alpha \beta]}^{\mathrm{tw} 2}\left(\kappa_{1} \tilde{x}, \kappa_{2} \tilde{x}\right)= & -2 \int_{0}^{1} \mathrm{~d} \lambda(1-\lambda)\left\{\mathrm{d}_{[\alpha} \delta_{\beta]}^{[\mu} \tilde{x}^{\nu]}+X_{[\alpha \beta]} X^{[\mu \nu]}\right\} M_{[\mu \nu]}\left(\kappa_{1} \lambda \tilde{x}, \kappa_{2} \lambda \tilde{x}\right) \\
M_{[\alpha \beta]}^{\mathrm{tw} 3}\left(\kappa_{1} \tilde{x}, \kappa_{2} \tilde{x}\right)= & \int_{0}^{1} \mathrm{~d} \lambda \frac{1-\lambda^{2}}{\lambda}\left\{\left((\tilde{x} \mathrm{~d}) \delta_{[\alpha}^{[\mu} \delta_{\beta]}^{\nu]}-2 \delta_{[\alpha}^{[\mu} \tilde{x}^{\nu]} \mathrm{d}_{\beta]}\right)\right. \\
& -\int_{0}^{1} \mathrm{~d} \lambda \frac{1-\lambda^{2}}{\lambda} X_{[\alpha \beta]} X^{[\mu \nu]} M_{[\mu \nu]}\left(\kappa_{1} \lambda \tilde{x}, \kappa_{2} \lambda \tilde{x}\right) \\
M_{[\alpha \beta]}^{\mathrm{tw} 4}\left(\kappa_{1} \tilde{x}, \kappa_{2} \tilde{x}\right)= & 2 \int_{0}^{1} \mathrm{~d} \lambda \frac{1-\lambda}{\lambda}\left\{X_{[\alpha \beta]} X^{[\mu \nu]}-\tilde{x}_{[\alpha} \delta_{\beta]}^{[\mu} \mathrm{d}^{\nu]}\right\} M_{[\mu \nu]}\left(\kappa_{1} \lambda \tilde{x}, \kappa_{2} \lambda \tilde{x}\right) \\
M_{\alpha}^{\mathrm{tw} 2}\left(\kappa_{1} \tilde{x}, \kappa_{2} \tilde{x}\right)= & -\int_{0}^{1} \mathrm{~d} \lambda \lambda \ln \lambda\left\{X^{2} \delta_{\alpha}^{\mu}-\tilde{x}_{\alpha} \mathrm{d}^{\mu}\right\} M_{\mu}\left(\kappa_{1} \lambda \tilde{x}, \kappa_{2} \lambda \tilde{x}\right) \\
M_{\alpha}^{\mathrm{tw} 3}\left(\kappa_{1} \tilde{x}, \kappa_{2} \tilde{x}\right)= & -\int_{0}^{1} \mathrm{~d} \lambda \lambda \ln \lambda \tilde{x}_{\alpha} \mathrm{d}^{\mu} M_{\mu}\left(\kappa_{1} \lambda \tilde{x}, \kappa_{2} \lambda \tilde{x}\right) .
\end{aligned}
$$


Let us mention that the actual tensor and vector LC-operators satisfy conditions of tracelessness on the light-cone which can be formulated by means of the interior derivative $\mathrm{d}_{\mu}$ [29]. For example, these conditions simply read (see [30,2]):

$$
\mathrm{d}^{\alpha} O_{\alpha}^{\tau=2,3}\left(\kappa_{1} \tilde{x}, \kappa_{2} \tilde{x}\right)=0, \quad \mathrm{~d}^{\alpha} M_{[\alpha \beta]}^{\tau=2}\left(\kappa_{1} \tilde{x}, \kappa_{2} \tilde{x}\right)=0, \quad \mathrm{~d}^{\alpha} M_{\alpha}^{\tau=2}\left(\kappa_{1} \tilde{x}, \kappa_{2} \tilde{x}\right)=0 .
$$

Scalar operators on the light-cone are traceless by construction since they correspond to totally symmetrized tensors.

These relations may be proven very easily by using the above mentioned properties of inner derivatives. Also the proof of the projection properties is straightforward but tedious.

Let us also rewrite the local twist operators $(2.22)-(2.29)$ by means of inner derivatives:

$$
\begin{aligned}
O_{\alpha n}^{\mathrm{tw} 2}(\tilde{x})= & \frac{1}{(n+1)^{2}} \mathrm{~d}_{\alpha} O_{n+1}(\tilde{x}) \\
O_{\alpha n}^{\mathrm{tw} 3}(\tilde{x})= & \frac{1}{(n+1) n}\left(n^{2} \delta_{\alpha}^{\mu}-\tilde{x}^{\mu} \mathrm{d}_{\alpha}-\frac{n-1}{n+1} \tilde{x}_{\alpha} \mathrm{d}^{\mu}\right) O_{\mu n}(\tilde{x}) \\
O_{\alpha n}^{\mathrm{tw} 4}(\tilde{x})= & \frac{1}{(n+1)^{2}} \tilde{x}_{\alpha} \mathrm{d}^{\mu} O_{\mu n}(\tilde{x}) \\
M_{[\alpha \beta] n}^{\mathrm{tw} 2}(\tilde{x})= & \frac{2}{(n+2)(n+1)}\left\{\mathrm{d}_{[\beta} \delta_{\alpha]}^{\mu}-\frac{1}{n+2} \tilde{x}_{[\alpha} \tilde{\partial}_{\beta]} \mathrm{d}^{\mu}\right\} M_{\mu n+1}(\tilde{x}) \\
M_{[\alpha \beta] n}^{\mathrm{tw} 3}(\tilde{x})= & \frac{1}{(n+2) n}\left\{\delta_{[\alpha}^{\mu} \delta_{\beta]}^{\nu} n^{2}-2 \tilde{x}^{\nu} \delta_{[\alpha}^{\mu} \mathrm{d}_{\beta]}+\frac{2}{n} \tilde{x}_{[\alpha}\left(\delta_{\beta]}^{\mu}(n-1)-\tilde{x}^{\mu} \tilde{\partial}_{\beta]}\right) \mathrm{d}^{\nu}\right\} M_{[\mu \nu] n}(\tilde{x}) \\
& +\frac{2}{(n+2)^{2} n} \tilde{x}_{[\alpha} \tilde{\partial}_{\beta]} \mathrm{d}^{\mu} M_{\mu n+1}(\tilde{x}) \\
M_{[\alpha \beta] n}^{\mathrm{tw} 4}(\tilde{x})= & \frac{2}{(n+1) n}\left\{\tilde{x}_{[\alpha} \tilde{\partial}_{\beta]} \tilde{x}^{[\mu} \tilde{\partial}^{\nu]}-\tilde{x}_{[\alpha} \delta_{\beta]}^{[\mu} \mathrm{d}^{\nu]}\right\} M_{[\mu \nu] n}(\tilde{x}) \\
M_{\alpha n+1}^{\mathrm{tw} 2}(\tilde{x})= & M_{\alpha n+1}(\tilde{x})-\frac{1}{(n+2)^{2}} \tilde{x}_{\alpha} \mathrm{d}^{\mu} M_{\mu n+1}(\tilde{x}) \\
M_{\alpha n+1}^{\mathrm{tw} 3}(\tilde{x})= & \frac{1}{(n+2)^{2}} \tilde{x}_{\alpha} \mathrm{d}^{\mu} M_{\mu n+1}(\tilde{x}) .
\end{aligned}
$$

Now we point out some technical details. The local operators of definite twist are written in such a manner that their relation to the various symmetry classes by which they are determined becomes more obvious (for details see [1.2]). The twist-4 operator (A.19) is built up by those parts of the operators (A.17) and (A.18) which are related to the Young pattern $(n-1)$, i.e., being contained in the trace terms. The twist-3 operator (A.21) consists of two independent operators, namely a twist -3 operator corresponding to the traces of the Young pattern $(n, 2)$ which obey the totally symmetric pattern $(n)$, and another operator which is constructed by means of the Young pattern $(n, 1,1)$. Both terms have been separately written, cf. also Eqs. (A.11) and (A.12). The twist-4 operator (A.22) contains the $(n-1,1)$ part of the traces of the Young patterns $(n, 2)$ and $(n, 1,1)$.

Let us discuss the conditions of tracelessness for the local light-cone operators in more detail. For the leading twist operators these conditions are

$$
\mathrm{d}^{\alpha} O_{\alpha n}^{\tau=2,3}(\tilde{x})=0, \quad \mathrm{~d}^{\alpha} M_{[\alpha \beta] n}^{\tau=2}(\tilde{x})=0, \quad \mathrm{~d}^{\alpha} M_{\alpha n+1}^{\tau=2}(\tilde{x})=0 .
$$

Properly speaking, the higher twist parts of the vector operators $O_{\alpha n+1}^{\mathrm{tw} 4}(\tilde{x})$ and $M_{\alpha n+1}^{\mathrm{tw} 3}(\tilde{x})$ are $\mathrm{d}^{\mu} O_{\mu n}(\tilde{x})$ and $\mathrm{d}^{\mu} M_{\mu n+1}(\tilde{x})$, respectively. In fact, these operators are scalar operators being traceless on the light-cone. The case of the higher twist tensor operators is more complicated. The condition of tracelessness for the twist-3 tensor operator corresponding to the pattern $(n, 1,1)$, i.e. the first term in (A.21), reads

$$
\mathrm{d}^{\alpha} M_{[\alpha \beta] n}^{\tau=3}(\tilde{x})=0
$$

Actually, the other term is a twist-3 vector operator $\widetilde{M}_{\beta n-1}^{\mathrm{tw} 3}(\tilde{x})$ multiplied by $\tilde{x}_{\alpha}$,

$$
M_{[\alpha \beta] n}^{\mathrm{tw} 3}(\tilde{x})=\frac{2}{(n+2)^{2} n^{2}} \tilde{x}_{[\alpha} \widetilde{M}_{\beta] n-1}^{\mathrm{tw} 3}(\tilde{x}), \quad \widetilde{M}_{\beta n-1}^{\mathrm{tw} 3}(\tilde{x})=\mathrm{d}_{\beta} \mathrm{d}^{\mu} M_{\mu n+1}(\tilde{x}),
$$

and also the twist- 4 tensor operator is given analogously by a vector operator $\widetilde{M}_{\beta n}^{\text {tw }}(\tilde{x})$,

$$
M_{[\alpha \beta] n}^{\mathrm{tw} 4}(\tilde{x})=\frac{2}{(n+1) n} \tilde{x}_{[\alpha} \widetilde{M}_{\beta] n-1}^{\mathrm{tw} 4}(\tilde{x}), \quad \widetilde{M}_{\beta n-1}^{\mathrm{tw} 4}(\tilde{x})=\left\{\frac{1}{n} \mathrm{~d}_{\beta} \tilde{x}^{[\mu} \tilde{\partial}^{\nu]}-\delta_{\beta}^{[\mu} \mathrm{d}^{\nu]}\right\} M_{[\mu \nu] n}(\tilde{x}) .
$$


The conditions of tracelessness for these vector operators read

$$
\mathrm{d}^{\beta} \widetilde{M}_{\beta n-1}^{\mathrm{tw} 3}(\tilde{x})=0, \quad \mathrm{~d}^{\beta} \widetilde{M}_{\beta n-1}^{\mathrm{tw} 4}(\tilde{x})=0 .
$$

It is clear that after multiplication with $\tilde{x}_{\alpha}$ and antisymmetrization with respect to $\alpha$ and $\beta$ the trace terms of these vector operators are cancelled.

Each series of local operators (A.17) - A.24 may be re-expressed by one nonlocal operator by using formulas like $\kappa^{n} /(n+1)^{2}=-\int_{0}^{1} \mathrm{~d} \lambda(\ln \lambda)(\kappa \lambda)^{n}$ as easily can be seen in the case of twist -2 vector operators. The other fractions are somewhat more involved; factors of $n$ in the nominator are to be expressed by $(\tilde{x} \tilde{\partial})$.

The expressions for the twist $-\tau$ operators in terms of internal derivatives is not unique because, by using formulas like $\tilde{x}^{[\mu} \mathrm{d}^{\nu]}=(\tilde{x} \tilde{\partial}) \tilde{x}^{[\mu} \tilde{\partial}^{\nu]}$ and performing partial $\lambda$-integrations, they may be reformulated. This has been done for the nonlocal operators A.7) - A.15) at various places in order to obtain a form where their projection properties can be proven most easily by using the commutation relations between the generators $\tilde{x}_{\mu}, \mathrm{d}_{\mu}, X$ and $X_{\mu \nu}$. Obviously, some of these reformulations are true only in the case of the antisymmetric tensor operator whereas the corresponding local expressions hold more generally.

Finally, let us mention that Dobrev et al. 28, 31] used the interior derivative $\mathrm{d}_{\mu}$ for the construction of local symmetric (traceless) light-cone operators which carry an elementary (irreducible) representation of the conformal group $S O(4,2)$.

\section{APPENDIX B: TRANSFORMATION PROPERTIES OF NONLOCAL LC OPERATORS}

Because the notion of geometric twist is related to the framework of the group $S O(3,1) \otimes \mathbb{R}_{+}$, where $\mathbb{R}_{+}$is the group of dilations, it is useful to give the transformation rules of the (unrenormalized) nonlocal operators with respect to this group. We induce the transformation rules of the nonlocal operators from the transformation properties of local fields which are given in [32,28]. Let $\mathcal{O}_{\alpha n}^{\tau}(y, \tilde{x})$ be the local components - being obtained by a formal Taylor expansion around the point $y$ - of any nonlocal operator $\mathcal{O}_{\alpha}^{\tau}(y-\tilde{x}, y+\tilde{x})\left(\right.$ with $\left.\kappa_{1}=-1, \kappa_{2}=1\right)$ of geometric twist $\tau$. These local operators $\mathcal{O}_{\alpha n}^{\tau}(y, \tilde{x})$ are infinitely differentiable with respect to $y \in \mathbb{R}^{4}$ and homogeneous polynomials of degree $n$ in the light-like vector $\tilde{x}$.

The transformation properties of these local operators are 32,28

a) Poincaré transformations:

$$
\hat{U}(a, \Lambda) \mathcal{O}_{\alpha n}^{\tau}(y, \tilde{x}) \hat{U}^{-1}(a, \Lambda)=V_{\alpha \beta}(\Lambda) \mathcal{O}_{\beta n}^{\tau}\left(\Lambda^{-1}(y-a), \Lambda^{-1} \tilde{x}\right), \quad a \in \mathbb{R}^{4}, \Lambda \in S O(3,1),
$$

where $V(\Lambda)$ is a finite dimensional representation of the Lorentz group. Obviously, the translation $\hat{U}(a, 0)=\mathrm{e}^{-\mathrm{i} a \cdot \hat{P}}$ with $\hat{P}_{\mu}=\mathrm{i} \frac{\partial}{\partial y^{\mu}}$ just shifts the expansion point $y$ by the vector $a$. If we would have chosen $a=\tilde{x}$, the twist of these operators would be not invariant under this translation, because the differential operators for definite twist do not commute with $\tilde{x}$.

b) Dilations:

$$
\hat{U}(\rho) \mathcal{O}_{\alpha n}^{\tau}(y, \tilde{x}) \hat{U}^{-1}(\rho)=\rho^{d} \mathcal{O}_{\beta n}^{\tau}(\rho y, \tilde{x}), \quad \rho>0,
$$

where $d$ is the (scale) dimension of the local operator (in mass units).

The transformation properties for the nonlocal operators are

a) Poincaré transformations:

$$
\hat{U}(a, \Lambda) \mathcal{O}_{\alpha}^{\tau}(y-\tilde{x}, y+\tilde{x}) \hat{U}^{-1}(a, \Lambda)=V_{\alpha \beta}(\Lambda) \mathcal{O}_{\beta}^{\tau}\left(\Lambda^{-1}(y-a-\tilde{x}), \Lambda^{-1}(y-a+\tilde{x})\right),
$$

b) Dilations:

$$
\hat{U}(\rho) \mathcal{O}_{\alpha}^{\tau}(y-\tilde{x}, y+\tilde{x}) \hat{U}^{-1}(\rho)=\rho^{d} \mathcal{O}_{\alpha}^{\tau}(\rho(y-\tilde{x}), \rho(y+\tilde{x})) .
$$

Let us point out that in the case nonlocal operators, i.e., for an infinite series of local ones, we deal with an infinitedimensional representation of the dilation and the Lorentz group. 
[1] B. Geyer, M. Lazar, and D. Robaschik, Nucl. Phys. B 559 (1999) 339.

[2] B. Geyer and M. Lazar, Nucl. Phys. B 581 (2000) 341.

[3] D.J. Gross and S.B. Treiman, Phys. Rev. D 4 (1971) 1059.

[4] R.L. Jaffe and X. Ji, Phys. Rev. Lett. 67 (1991) 552; Nucl. Phys. B 375 (1992) 527.

[5] P. Ball, V.M. Braun, Y. Koike, and K. Tanaka, Nucl. Phys. B 529 (1998) 323.

[6] P. Ball and V.M. Braun, Nucl. Phys. B 543 (1999) 201.

[7] R.L. Jaffe and M. Soldate, Phys. Lett. B 105 (1981) 467; Phys. Rev. D 26 (1982) 49.

[8] R.L. Jaffe, Nucl. Phys. B 229 (1983) 205.

[9] A.V. Radyushkin, Phys. Lett. B 380 (1996), 417; Phys. Rev. D 56 (1997) 5524.

[10] J. Blümlein, B. Geyer, and D. Robaschik, Nucl. Phys. B 560 (1999) 283.

[11] J. Blümlein and D. Robaschik, Nucl. Phys. B 581 (2000) 449.

[12] B. Ehrnsperger, L. Mankiewicz, and A. Schäfer, Phys. Lett. B 323 (1994) 439.

[13] M. Maul, B. Ehrnsperger, E. Stein, and A. Schäfer, Z. Phys. A 356 (1997) 443.

[14] A. Piccione and C. Ridolfi, Nucl. Phys. B 513 (1998) 301.

[15] J. Blümlein and A. Tkabladze, Nucl. Phys. B 553 (1999) 427.

[16] A.H. Guth and D.E. Soper, Phys. Rev. D 12 (1975) 1143.

[17] H. Georgi and H.D. Politzer, Phys. Rev. D 14 (1976) 1829.

[18] D. Müller, D. Robaschik, B. Geyer, F.-M. Dittes, and J. Hořejši, Fortsch. Phys. 42 (1994) 101.

[19] Y. Koike and K. Tanaka, Phys. Rev. D 51 (1995) 6125.

[20] J. Kodaira and K. Tanaka, Prog. Theor. Phys 101 (1999) 191.

[21] S. Wandzura and F. Wilczek, Phys. Lett. B 72 (1977) 195.

[22] J. Blümlein and N. Kochelev, Nucl. Phys. B 498 (1997) 285.

[23] M. Lazar, Konstruktion und Anwendung irreduzibler nichtlokaler Lichtkegeloperatoren in der Quantenchromodynamik, Diplomarbeit, Leipzig 1998.

[24] H. Burkhardt and W.N. Cottingham, Ann. Phys. (N.Y.) 56 (1970) 453.

[25] M. Lazar, $\rho$-Meson wave functions from nonlocal light-cone operators with definite twist, hep-ph/0009311, Phys. Lett. B in print.

[26] M. Lazar, Wandzura-Wilczek-type relations of $\rho$-meson wave functions, to appear.

[27] V. Bargmann and I.T. Todorov, J. Math. Phys. 18 (1977) 1141.

[28] V.K. Dobrev, G. Mack, V.B. Petkova, S.G. Petrova, and I.T. Todorov, Harmonic Analysis of the n-Dimensional Lorentz Group and its Applications to Conformal Quantum Field Theory, Lecture Notes in Physics, No. 63 (Springer, 1977 ).

[29] V.K. Dobrev and A.Ch. Ganchev, Conformal operators from spinor fields: Antisymmetric tensor case, Dubna preprint E2-82-881 (1982).

[30] B. Geyer and M. Lazar, Twist decomposition of nonlocal light-ray operators and harmonic tensor functions, Proc. Int. Conf. "Quantum Theory and Symmetries", Eds.: H.-D. Doebner, V.K. Dobrev, J.-D. Hennig and W. Lücke (World Scientific, Singapore, 2000) p. 362.

[31] N.S. Craigie, V.K. Dobrev, and I.T. Todorov, Ann. Phys. (N.Y.) 159 (1985) 411.

[32] V.K. Dobrev, G. Mack, V.B. Petkova, S.G. Petrova, and I.T. Todorov, Rep. Math. Phys. 9 (1976) 219. 